\title{
Scaling limits and regularity results for a class of Ginzburg-Landau systems
}

by

\author{
Robert L. JERRARD * \\ Department of Mathematics University of Illinois \\ 1409 West Green Street Urbana, IL 61801, USA \\ and \\ Halil Mete SONER ${ }^{\dagger}$ \\ Department of Mathematics Carnegie Mellon University Pittsburgh, PA 15213, USA
}

ABSTRACT. - We study a class of parabolic systems which includes the Ginzburg-Landau heat flow equation,

$$
u_{t}^{\epsilon}-\Delta u^{\epsilon}+\frac{1}{\epsilon^{2}}\left(\left|u^{\epsilon}\right|^{2}-1\right) u^{\epsilon}=0
$$

for $u^{\epsilon}: \mathbf{R}^{d} \rightarrow \mathbf{R}^{2}$, as well as some natural quasilinear generalizations for functions taking values in $\mathbf{R}^{k}, k \geq 2$.

We prove that for solutions of the general system, the limiting support as $\epsilon \rightarrow 0$ of the energy measure is a codimension $k$ manifold which evolves via mean curvature.

We also establish some local regularity results which hold uniformly in $\epsilon$. In particular, we establish a small-energy regulity theorem for the general system, and we prove a stronger regularity result for the usual Ginzburg-Landau equation on $\mathbf{R}^{2}$.

(C) 1999 L'Association Publications de l'Institut Henri Poincaré. Published by Elsevier B.V. All rights reserved

\footnotetext{
* Partially supported by the Army Research Office and the National Science Foundation through the Center for Nonlinear Analysis and by the NSF grant DMS-9200801.

${ }^{\dagger}$ Partially supported by the Army Research Office and the National Science Foundation through the Center for Nonlinear Analysis and by the NSF grants DMS-9200801, DMS-9500940, and by the ARO grant DAAH04-95-1-0226.
}

Annales de l'Institut Henri Poincaré - Analyse non linéaire - 0294-1449 
RÉSUMÉ. - Nous étudions une classe de systèmes paraboliques qui comprennent l'equation de chaleur Ginzburg-Landau,

$$
u_{t}^{\epsilon}-\Delta u^{\epsilon}+\frac{1}{\epsilon^{2}}\left(\left|u^{\epsilon}\right|^{2}-1\right) u^{\epsilon}=0
$$

pour $u^{\epsilon}: \mathbf{R}^{d} \rightarrow \mathbf{R}^{2}$, ainsi que des généralisations quasilinéaires pour des fonctions prenant leurs valeurs dans $\mathbf{R}^{k}, k \geq 2$.

Nous prouvons que, pour les solutions du système général, le support limite (lorsque $\epsilon \rightarrow 0$ ) de mèsure d'énergie est une varieté de codimension $k$ qui évolue selon sa courbure moyenne.

Nous établissons en addition quelques resultats de regularité locale, qui sont valides uniformement en $\epsilon$.

(C) 1999 L'Association Publications de l'Institut Henri Poincaré. Published by Elsevier B.V. All rights reserved

\section{INTRODUCTION}

We present in this paper a collection of results concerning the asymptotic regularity and qualitative behavior of solutions of the Ginzburg-Landau system,

$$
\begin{gathered}
u_{t}^{\epsilon}-\Delta u^{\epsilon}+\frac{1}{\epsilon^{2}}\left(\left|u^{\epsilon}\right|^{2}-1\right) u^{\epsilon}=0 \quad \text { in } \mathbf{R}^{d} \times[0, T], \\
u^{\epsilon} \in \mathbf{R}^{2}
\end{gathered}
$$

We also propose and study a class of equations which we believe are natural generalizations of (1.1). These systems have the form

$$
\begin{gathered}
u_{t}^{\epsilon}-\Delta u^{\epsilon}-\frac{p-2}{2} \frac{\nabla\left[e_{\epsilon}\left(u^{\epsilon}\right)\right] \cdot \nabla u^{\epsilon}}{e_{\epsilon}\left(u^{\epsilon}\right)}+\frac{1}{\epsilon^{2}}\left(\left|u^{\epsilon}\right|^{2}-1\right) u^{\epsilon}=0 \quad \text { in } \mathbf{R}^{d} \times[0, T], \\
u^{\epsilon} \subset \mathbf{R}^{k}, \quad k \geq 2
\end{gathered}
$$

Here

$$
e_{\epsilon}(u):=\frac{|\nabla u|^{2}}{2}+\frac{1}{\epsilon^{2}} W(u), \quad W(u):=\frac{1}{4}\left(|u|^{2}-1\right)^{2} .
$$

Of special interest is the case where $p=k$; this is a direct generalization of (1.1). 
The Ginzburg-Landau system arises in a variety of contexts, including models of superconductivity and of systems of coupled oscillators near a bifurcation point, see for example Kuramoto [27]. Recently the associated minimization problem has been studied in great detail by Betheul, Brezis, and Hélein [4], [5], with refinements by Struwe [26], among others. Neu [19], Pismen and Rubinstein [20], Rubinstein [21], E [9], and others have analysed (1.1) and the associated Schroedinger-type equation using matched asymptotic expansions. A number of results on the behavior of (1.1) in two space dimensions were obtained by Lin [17], [18].

We view (1.2) as a natural generalization of (1.1) to energies with nonquadratic growth in the gradient term. Given a solution $u^{\epsilon}$ of (1.2) we define

$$
E^{\epsilon}\left(u^{\epsilon}\right)=\frac{2}{p} e_{\epsilon}\left(u^{\epsilon}\right)^{p / 2} .
$$

We think of $E^{\epsilon}$ as a energy density for the generalized Ginzburg-Landau system. This interpretation is motivated by the fact that

$$
I^{\epsilon}\left(u^{\epsilon}\right)=\int E^{\epsilon}\left(u^{\epsilon}\right) d x
$$

is formally a Lyapunov functional for (1.2). We remark that (1.2) is not an equation for gradient flow for the functional $I^{\epsilon}$. However, it retains many of the estimates satisfied by (1.1), estimates which are crucial to any analysis of properties of solutions. (These estimates are chiefly presented in Section 2).

Also, in the same way that (1.1) is a kind of model problem for codimension 2 pattern formation, the generalized system (1.2) can serve as a model problem for the study of higher codimension pattern formation. This view is supported by the results we present in Section 3, which are discussed immediately below.

Our results fall into two classes. First, we characterize the qualitative behavior of solutions of (1.2) in the limit as $\epsilon \rightarrow 0$, in the case where $d>k=p$. More precisely, given a family of solutions $u^{\varepsilon}$ of (1.2) with appropriate initial data, we define an associated family of measures $\nu_{t}^{\epsilon}$, and we show that the support of these measures, in the limit, forms exactly a $(d-k)$-dimensional submanifold which evolves via codimension $k$ mean curvature flow, at least for short times.

This result, which occupies Section 3, confirms the formal computations of Rubinstein [21], Pismen and Rubinstein [20], and E [9] for the usual Ginzburg-Landau system (1.1) in three space dimensions, and also applies 
to more general situations. It is closely related to a number of recent results about the asymptotic behavior of solutions of scalar Ginzburg-Landau equations and related equations. For example, Chen [7], Evans, Soner and Souganidis [11], Ilmanen [23], and Soner [23] have shown that solutions of the Allen-Cahn equation in a singular limit exhibit a sharp interface which evolves via codimension 1 mean curvature flow. The latter three papers establish this result globally in time, using various weak notions of evolution via mean curvature. Analagous results have been established for more general scalar reaction-diffusion equations by Barles, Soner and Souganidis [2] and Jerrard [12], among others.

The larger part of this paper is devoted to establishing some regularity theorems. We first prove a small energy regularity result. In Section 4 we prove that if certain weighted integrals of the energy density $E^{*}$ are sufficiently small, then $E^{t}$ is in fact bounded in some smaller region. This result is valid uniformly for parameter values $\epsilon \in(0.1]$. Our proof uses a monotonicity formula and a Bochner inequality, following ideas of Struwe [24], and Chen and Struwe [8]. Small energy regularity and a covering argument imply partial regularity results, as in Chen and Struwe [8].

In the special case of the usual Ginzburg-Landau equation in $\mathbf{R}^{2} \times[0, T]$, we establish much stronger regularity results. We prove that if integrals of the energy density are bounded in some region, then in fact the energy is pointwise bounded in a smaller region. This result, which is again uniform in $\epsilon$, follows from the small energy regularity via a blowup argument (Section 6) and a Liouville-type theorem (Section 7). The blowup argument is similar to one found in Struwe [25].

This latter regularity result is used in another paper by the authors, [14] in which we completely characterize the asymptotic behavior of solutions of (1.1) in $\Omega \times[0, T]$, where $\Omega \subset \mathbf{R}^{2}$ and $T>0$. This result, which is valid only locally in time, provides rigorous proof of formal results of Neu [19], E [9] and others.

The paper starts with a collection of estimates in Section 2.

One issue we do not address is the solvability of (1.2). It is wellknown that (1.1) admits smooth solutions; this follows from the work of Ladyzhenskaya, Solonnikov, and Uraltseva [16], as is verified in Bauman, Chen, Phillips, and Sternberg [3], for example. Results of this sort are not so obvious in the case of the generalized system (1.2). It is not difficult to construct some sort of weak solutions of (1.2), for example by discretizing in time, solving implicitly at each time step, and passing to limits. To establish regularity, however, seems to require a priori $C^{1, c}$ estimates. 
Such estimates are not, in general, valid for quasilinear systems, but they normally hold for systems for which there is some sort of energy density which is itself a subsolution of an elliptic or parabolic equation. This is the case for (1.2), as is shown in Proposition 2.1. It is therefore not unreasonable to expect that the same estimate holds for (1.2), and thus that smooth solutions exist. In this paper, however, we focus on other issues and simpy assume the existence of smooth solutions.

We will always assume that the initial data for (1.2) satisfies

$$
\left\|u^{\epsilon}(\cdot, 0)\right\|_{L^{\infty}} \leq 1, \quad\left\|E^{\epsilon}(\cdot, 0)\right\|_{L^{1}}<+\infty .
$$

Multiplying (1.2) by $u^{\epsilon}$ and defining $w^{\epsilon}:=\left|u^{\epsilon}\right|^{2}$, we discover

$$
w_{t}^{\epsilon}-\Delta w^{\epsilon}-\frac{p-2}{2 \epsilon_{\epsilon}} \nabla \epsilon \cdot \nabla w^{\epsilon}+\frac{2}{\epsilon^{2}}\left(w^{\epsilon}-1\right) w^{\epsilon}+2\left|\nabla u^{\epsilon}\right|^{2}=0 .
$$

The maximum principle thus suggests that any reasonable solution should satisfy

$$
\left|u^{\epsilon}(x, t)\right| \leq 1
$$

for all $(x, t) \in \mathbf{R}^{d} \times[0, \infty)$. Similarly, estimates in Section 2 imply that a well-behaved solution should have the property that

$$
\int_{\mathbf{R}^{d}} E^{\epsilon}(x, t) d x+\int_{0}^{t} \int_{\mathbf{R}^{d}} e_{\epsilon}^{\frac{p-2}{2}}(x, s)\left|u_{t}^{\epsilon}(x, s)\right|^{2} d x d s=\int_{\mathbf{R}^{d}} E^{\epsilon}(x, 0) d x
$$

Both of these statements will hold, roughly speaking, as long as there is no influx of energy from $|x|=+\infty$. It is not hard to see, for example, that a solution produced by the implicit time discretization described above will have these properties. We therefore further assume that for initial data as described, our solutions satisfy both (1.6) and (1.7). To establish these estimates a priori would require a delicate analysis and might not be possible, as is shown by the example of the heat equation.

\section{NOTATION AND PRELIMINARIES}

We will use the following notation throughout this paper.

Integers $d$ and $k$ will always denote the dimensions of the domain and the range, respectively, of the mappings we consider.

$e_{\epsilon}(\cdot)$ and $E^{\epsilon}(\cdot)$ will always be as defined in (1.2) and (1.3), where the power $p$ in the latter definition is understood to be the same as that in the Vol. $16, n^{\circ} 4-1999$. 
generalized system (1.2). We will normally write $e_{\epsilon}$ instead of $e_{\epsilon}\left(u^{\epsilon}\right)$, when no confusion can result, and likewise $E^{\epsilon}$.

We employ the summation convention throughout. Roman indices $i, j, \ldots$ are always understood to run from 1 to $d$, and greek indices $\alpha, \beta, \ldots$ run from 1 to $k$. Exceptions will be indicated explicitly. A scalar product between matrices is denoted by $A: B$, so that for example $\nabla u: \nabla v:=u_{x_{i}}^{\alpha} v_{x_{i}}^{\alpha}$.

We also use the notation

$$
B_{p}^{n}\left(x_{0}\right)=\left\{x \in \mathbf{R}^{n}|| x-x_{0} \mid \leq \rho\right\} .
$$

We will normally omit the superscript $n$ which indicates the dimension of the ambient space, displaying it only when the dimension is not obvious from the context.

Observe that if $u^{\epsilon}$ solves (1.2) for a given value of the parameter $\epsilon$, then $\tilde{u}(x, t):=u^{\epsilon}\left(\sigma x, \sigma^{2} t\right)$ solves (1.2) with $\tilde{e}:=\epsilon / \sigma$. Similarly, we have $E^{\tilde{e}}(\tilde{u})(x, t)=\sigma^{p} E^{\epsilon}\left(u^{\epsilon}\right)\left(\sigma x, \sigma^{2} t\right)$. Rescaling in this fashion, we can convert statements about solutions of (1.2) for arbitrary $\epsilon$ into statements about solutions with $\epsilon=1$, for example. Whenever a statement of a theorem is invariant under this rescaling, it clearly suffices to prove it for a single value of the parameter $\epsilon$. We will invoke this sort of argument from time to time by saying, without further explanation, that it suffices "by a rescaling argument" to consider a certain case.

\section{ESTIMATES}

In this section we collect some estimates that we will use throughout this paper.

We assume that $u^{\epsilon}$ is a smooth solution of (1.2) on $\mathbf{R}^{d} \times[0, \infty)$ and that $E^{\epsilon}(\cdot, 0) \in L^{1}\left(\mathbf{R}^{d}\right)$.

Following a suggestion of $M$. Grillakis we define

$$
\begin{gathered}
\mu_{t}^{\epsilon}(d x)=E^{\epsilon}(x, t) d x, \\
p^{\epsilon}=\left(e_{\epsilon}\right)^{\frac{p-2}{2}} \nabla u^{\epsilon} \cdot u_{t}^{\epsilon}, \\
\sigma^{\epsilon}=\left(e_{\epsilon}\right)^{\frac{p-2}{2}} \nabla u^{\epsilon} \otimes \nabla u^{\epsilon} .
\end{gathered}
$$

The following fundamental identities are immediate consequences of the equation (1.2). We have

$$
E_{t}^{\epsilon}=\operatorname{div} p^{\epsilon}-\left(e_{\epsilon}\right)^{\frac{p-2}{2}}\left|u_{t}^{\epsilon}\right|^{2}
$$




$$
\nabla F^{\epsilon}=\operatorname{div} \sigma^{\epsilon}-p^{\epsilon} .
$$

Given a smooth test function $\eta \in W^{2, \infty}\left(\mathbf{R}^{d} \times[0, \infty]\right)$, we multiply the first equation above by $\eta$ and the second by $\nabla \eta$, then subtract to obtain

$$
\eta E_{t}^{\epsilon}=\nabla \eta \cdot \nabla E^{\epsilon}+\operatorname{div}\left(\eta p^{\epsilon}\right)+\nabla \eta \cdot \operatorname{div} \sigma^{\epsilon}-\eta\left(e_{\epsilon}\right)^{\frac{p-2}{2}}\left|u_{t}^{\epsilon}\right|^{2} .
$$

We integrate to find

$$
\begin{aligned}
\frac{d}{d t} \int \eta d \mu_{t}^{\epsilon}=-\int\left(e_{\epsilon}\right)^{\frac{p-2}{2}} \eta\left|u_{t}^{\epsilon}\right|^{2} d x+ & \int\left(\eta_{t}-\Delta \eta\right) d \mu_{t}^{\epsilon} \\
& +\int\left(e_{\epsilon}\right)^{\frac{p-2}{2}} \nabla^{2} \eta \nabla u^{\epsilon} \nabla u^{\epsilon} d x .
\end{aligned}
$$

By adding, rather than subtracting, equations (2.1) and (2.2), we obtain in a similar fashion

$$
\begin{aligned}
\frac{d}{d t} \int \eta d \mu_{\iota}^{\epsilon}=- & \int \eta\left(e_{\epsilon}\right)^{\frac{p-2}{2}}\left|u_{t}^{\epsilon}+\frac{\nabla u^{\epsilon} \cdot \nabla \eta}{\eta}\right|^{2} d x+\int\left(\eta_{t}+\Delta \eta\right) d \mu_{i}^{\epsilon} \\
& +\int\left(e_{\epsilon}\right)^{\frac{p-2}{2}}\left(\frac{\left|\nabla u^{\epsilon} \cdot \nabla \eta\right|^{2}}{\eta}-\nabla^{2} \eta \nabla u^{\epsilon} \nabla u^{\epsilon}\right) d x
\end{aligned}
$$

The integration by parts that we have carried out above is justified if

$$
\nabla \eta(\cdot, t) E^{\epsilon}(\cdot, t) \in L^{1}\left(\mathbf{R}^{d}\right), \quad \eta(\cdot, t) p^{\epsilon}(\cdot, t) \in L^{1}\left(\mathbf{R}^{d}\right) .
$$

The former follows from our standing assumption (1.7). Invoking the same assumption, the latter holds for a.e. $t$, since

$$
\int p^{\epsilon} d x \leq \int E^{\epsilon} d x+\int e_{\epsilon}^{\frac{p-2}{2}}\left|u_{t}^{\epsilon}\right|^{2} d x
$$

and the right-hand side is finite a.e. $t$. Whenever we apply the above estimates, we will integrate them over some time interval, so we can safely ignore the set of measure zero on which $p^{\epsilon}(\cdot, t)$ is not integrable.

We next show that the energy density $E^{\epsilon}$ solves a certain parabolic equation. In the statement and proof of this lemma we omit all superscripts $\epsilon$, and we write $e$ to mean $e(u)=e_{\epsilon}\left(u^{\circ}\right)$.

Proposition 2.1. - The energy density $E$ satisfies

$$
\begin{gathered}
E_{t}-\Delta E-\frac{p-2}{2 e} \nabla^{2} E \nabla u \cdot \nabla u=e^{\frac{p-2}{2}}\left[\frac{2}{\epsilon^{2}}\left(1-|u|^{2}\right)|\nabla u|^{2}-\left|\nabla^{2} u\right|^{2}\right. \\
\left.-\frac{p(p-2)}{4} \frac{|\nabla e \cdot \nabla u|^{2}}{e^{2}}-\frac{4}{\epsilon^{2}}|u \cdot \nabla u|^{2}-\frac{1}{\epsilon^{4}}\left(1-|u|^{2}\right)^{2}|u|^{2}\right]
\end{gathered}
$$


Also,

$E_{t}-\Delta E-\frac{p-2}{2 e} \nabla^{2} E \nabla u \cdot \nabla u+e^{\frac{p-2}{2}}\left[\left|\nabla^{2} u\right|^{2}+\frac{1}{2 \epsilon^{4}}\left(1-|u|^{2}\right)^{2}|u|^{2}\right] \leq C E^{\frac{p+2}{p}}$.

Proof. - From the definition of $E$ we compute

$$
E_{t}=e^{\frac{p-2}{2}}\left(\nabla u: \nabla u_{t}+\frac{1}{\epsilon^{2}}\left(|u|^{2}-1\right) u \cdot u_{t}\right)
$$

We now replace $u_{t}$ and $\nabla u_{t}$ in the above equation by expressions we obtain from the generalized Ginzburg-Landau system (1.2), thereby obtaining

$$
\begin{gathered}
E_{t}=e^{\frac{p-2}{2}}\left[\nabla u: \nabla \Delta u+\frac{p-2}{2}\left(\frac{u_{x_{i}}^{\alpha} u_{x_{j}}^{\alpha} e_{x_{i} x_{j}}}{e}+\frac{u_{x_{j}}^{\alpha} u_{x_{i} x_{j}}^{\alpha} e_{x_{i}}}{e}-\frac{|\nabla e \cdot \nabla u|^{2}}{e^{2}}\right)\right. \\
-\frac{2}{\epsilon^{2}}|u \cdot \nabla u|^{2}-\frac{\left(|u|^{2}-1\right)^{2}|u|^{2}}{\epsilon^{4}}+ \\
\left.\quad \frac{|u|^{2}-1}{\epsilon^{2}}\left(u \cdot \Delta u-|\nabla u|^{2}+\frac{p-2}{2} e_{x_{i}} u_{x_{j}}^{\alpha} u^{\alpha}\right)\right]
\end{gathered}
$$

(We have written out explicitly the terms for which there is some chance that more condensed notation might be ambiguous.) We also have

$$
\nabla^{2} E=e^{p-\frac{2}{2}}\left(\nabla^{2} e+\frac{p-2}{2} \frac{\nabla e \otimes \nabla e}{e}\right),
$$

from which we deduce that

$$
\begin{aligned}
\Delta E=e^{\frac{p-2}{2}}\left(\Delta e+\frac{p-2}{2} \frac{|\nabla e|^{2}}{e}\right) & \\
=e^{\frac{p-2}{2}} & {\left[\left|\nabla^{2} u\right|^{2}+\nabla u: \nabla \Delta u+\frac{2}{\epsilon^{2}}|u \cdot \nabla u|^{2}\right.} \\
& \left.\quad+\frac{\left(|u|^{2}-1\right)}{\epsilon^{2}}\left(|\nabla u|^{2}+u \cdot \nabla u\right)+\frac{p-2}{2} \frac{|\nabla e|^{2}}{e}\right] .
\end{aligned}
$$

From these we obtain, after cancelling several terms and combining terms of the same form,

$$
\begin{aligned}
& E_{t}-\Delta E-\frac{p-2}{2} \frac{\nabla u \cdot \nabla u \nabla^{2} E}{e}= \\
& \epsilon^{\frac{p-2}{2}}\left[-\left|\nabla^{2} u\right|^{2}-\frac{p(p-2)}{4} \frac{|\nabla e \cdot \nabla u|^{2}}{e^{2}}-\frac{4}{\epsilon^{2}}|u \cdot \nabla u|^{2}\right. \\
&-\frac{\left(|u|^{2}-1\right)^{2}|u|^{2}}{\epsilon^{4}}+\frac{2}{\epsilon^{2}}\left(1-|u|^{2}\right)|\nabla u|^{2} \\
&\left.+\frac{p-2}{2 e}\left(\nabla e \cdot \nabla\left(\frac{1}{2}|\nabla u|^{2}\right)-|\nabla e|^{2}+\frac{\left(|u|^{2}-1\right)}{\epsilon^{2}} e_{x_{i}} u_{x_{i}}^{\alpha} u^{\alpha}\right)\right] .
\end{aligned}
$$


From the definition of $e$ we see that

$$
\nabla e \cdot \nabla\left(\frac{1}{2}|\nabla u|^{2}\right)+\frac{\left(|u|^{2}-1\right)}{c^{2}} \nabla e \cdot \nabla\left(\frac{1}{2}|u|^{2}\right)-|\nabla e|^{2}=0 .
$$

The above two equations immediately imply that (2.5) holds.

To prove (2.6) from (2.5), note that Cauchy's inequality gives

$\frac{2}{\epsilon^{2}}\left(1-|u|^{2}\right)|\nabla u|^{2}-\frac{1}{\epsilon^{4}}\left(1-|u|^{2}\right)^{2}|u|^{2} \leq \frac{1}{2 \epsilon^{4}}\left(1-|u|^{2}\right)^{2}\left(\frac{1}{2}-|u|^{2}\right)+4|\nabla u|^{4}$.

If $|u|^{2} \geq 1 / 2$ then the first term in the right-hand side is negative. If, on the other hand, $|u|^{2} \leq 1 / 2$ then $\left(1-|u|^{2}\right)^{2} \leq 4\left(1-|u|^{2}\right)^{4}$. Therefore

$$
\begin{aligned}
\epsilon^{2}\left(1-|u|^{2}\right)|\nabla u|^{2}-\frac{1}{2 \epsilon^{4}}\left(1-|u|^{2}\right)^{2}|u|^{2} & \leq \frac{1}{\epsilon^{4}}\left(1-|u|^{2}\right)^{4}+4|\nabla u|^{4} \\
& \leq C e(u)^{2} .
\end{aligned}
$$

With (2.5) this immediately yields (2.6).

Finally, we derive some $L^{\infty}$ bounds for the energy. As these bounds depend on $\epsilon$, we again indicate explicitly the parameter $\epsilon$ in what follows.

From (2.5) we easily see that $w(x, t):=e^{-C t / \epsilon^{2}} E^{\epsilon}(x, t)$ satisfies

$$
w_{t}^{\epsilon}-\Delta w^{\epsilon}-\frac{p-2}{2 e} \nabla^{2} w^{\epsilon} \nabla u^{\epsilon} \cdot \nabla u^{\epsilon} \leq 0
$$

Thus the maximum principle implies that for any smooth solution $u^{\epsilon}$ and for all $s, t>0$,

$$
\|E(\cdot, s+t)\|_{\infty} \leq e^{C t / \epsilon^{2}}\|E(\cdot, s)\|_{\infty}
$$

If we strengthen our assumptions on the initial data, we obtain the following more useful result.

Proposition 2.2. - Let $u^{\epsilon} \in C^{\infty}\left(\mathbf{R}^{d} \times\left[0, I^{\prime}\right] ; \mathbf{R}^{k}\right)$ be a smooth solution of (1.2) with $p>2$, such that

$$
\sup _{x}\left[E^{\epsilon}(x, 0)+\frac{\kappa}{\epsilon^{p}}\left(\left|u^{\epsilon}(x, 0)\right|^{2}-1\right)\right] \leq 0
$$

for some $\kappa>0$. Then $\left\|E^{\epsilon}\right\|_{\infty} \leq C / \epsilon^{p}$.

Remark. - The conclusion of the lemma follows easily from standard regularity theory if $p=2$.

Proof. -1 . By rescaling it suffices to consider the case $\epsilon=1$. 
Let $w:=|u|^{2}$ and $\psi:=E+K(w-1)$, where $K \geq \kappa$ will be fixed below. For a smooth function $\phi$ let

$$
\mathcal{L} \phi:=\phi_{t}-\Delta \phi-\frac{p-2}{2 e} \nabla^{2} \phi \nabla u \cdot \nabla u
$$

Then using (1.5) we compute that

$$
\mathcal{L} w=2 w(1-w)-2|\nabla u|^{2}+\frac{p-2}{2 e} \nabla e \cdot \nabla w-\frac{p-2}{2 e} \nabla^{2} w \nabla u \cdot \nabla u .
$$

This with (2.5) gives

$$
\mathcal{L} \psi \leq-e^{(p-2) / 2}\left|\nabla^{2} u\right|^{2}+I_{1}+I_{2}
$$

where

$$
\begin{aligned}
& I_{1}=2 e^{(p-2) / 2}(1-w)|\nabla u|^{2}+2 K w(1-w)-2 K|\nabla u|^{2}-e^{(p-2) / 2}(1-w)^{2} w \\
& I_{2}=K \frac{p-2}{2 e} \nabla e \cdot \nabla w-K \frac{p-2}{2 e} \nabla^{2} w \nabla u \cdot \nabla u .
\end{aligned}
$$

2. We estimate

$$
\begin{aligned}
I_{1} & =-2 w\left[e^{\frac{p-2}{2}}\left(|\nabla u|^{2}+2 W(u)\right)-K(1-w)\right]+2 e^{\frac{p-2}{2}}|\nabla u|^{2}-2 K|\nabla u|^{2} \\
& =-2 w\left[2 e^{p / 2}+K(w-1)\right]+2|\nabla u|^{2}\left[e^{(p-2) / 2}-K\right] \\
& =-2 w \psi-2 w e^{p / 2}\left(2-\frac{2}{p}\right)+2|\nabla u|^{2}\left[e^{(p-2) / 2}-K\right] \\
& \leq 0
\end{aligned}
$$

on $\{\psi \geq 0\} \cap\left\{e^{(p-2) / 2}<K\right\}$. Also,

$$
\begin{aligned}
I_{2} & =\frac{K(p-2)}{2 e}\left[2 \nabla^{2} u^{\alpha} \nabla u^{\alpha} \cdot \nabla u^{\beta} u^{\beta}+(1 / 2)(w-1)|\nabla w|^{2}\right. \\
& \leq \frac{K(p-2)}{2 e}\left[4\left|\nabla^{2} u\right||\nabla u|^{2}-2|\nabla u|^{4}\right] .
\end{aligned}
$$

Hence

$$
\begin{aligned}
I_{2} & \leq \frac{2 K(p-2)}{e}\left|\nabla^{2} u\right||\nabla u|^{2} \\
& =e^{(p-2) / 2}\left|\nabla^{2} u\right|^{2}-e^{(p-2) / 2}\left(\left|\nabla^{2} u\right|-\frac{K(p-2)}{e^{p / 2}}|\nabla u|^{4}\right. \\
& -e^{(p-2) / 2} \frac{K(p-2)}{e^{p / 2}}|\nabla u|^{4}\left(1-\frac{K(p-2)}{e^{p / 2}}\right) \\
& \leq e^{(p-2) / 2}\left|\nabla^{2} u\right|^{2}
\end{aligned}
$$


on the set $\left\{e^{p / 2} \geq K(p-2)\right\}$. Combining these calculations, we obtain

$$
\mathcal{L} \psi \leq 0 \quad \text { on }\{\psi \geq 0\} \cap\left\{e^{(p-2) / 2} \leq K\right\} \cap\left\{e^{p / 2} \geq K(p-2)\right\} .
$$

Note that if $\psi \geq 2 K$ then $E \geq 2 K$ and thus $e^{p / 2} \geq K(p-2)$.

3. For $p>2$, set

$$
C(K, p):=\frac{2}{p} K^{p /(p-2)}-K .
$$

There is a $K(p) \geq 1$ such that $C(K, p) \geq 2 K+1$ for all $K \geq K(p)$. Moreover, if $K \geq K(p)$ and $\psi \leq 2 K+1$, then

$$
\begin{aligned}
\psi \leq C(K, p) & \Longrightarrow E \leq C(K, p)+K(1-w) \leq C(K, p)+K=\frac{2}{p} K^{p /(p-2)} \\
& \Longrightarrow e^{(p-2) / 2} \leq K .
\end{aligned}
$$

Therefore by taking $K=K(p) \vee \kappa$ in the definition of $\psi$, we get

$$
\mathcal{L} \psi \leq 0 \quad \text { on }\{2 K \leq \psi \leq 2 K+1\} .
$$

4. If we set $\phi:=\psi \vee 2 K$, then $\mathcal{L} \phi \leq 0$ on $\{\phi \leq 2 K+1\}$ (in the sense of viscosity solutions) and $\phi(x, 0) \equiv 2 K$. Let

$$
c(t):=\sup \left\{\phi(x, s) \mid x \in \mathbf{R}^{d}, s \in[0, t]\right\} .
$$

and define

$$
t_{0}:=\max \{t \geq 0 \mid c(t) \leq 2 K+1\} .
$$

From (2.7) we deduce that $c(\cdot)$ is continuous and that $t_{0}>0$. Also, $\mathcal{L} \phi \leq 0$ on $\mathbf{R}^{d} \times\left(0, t_{0}\right)$ and so the maximum principle implies that if $t<t_{0}$ then $\phi(x, t) \leq \phi(x, 0)=2 K$. Thus $t_{0}=+\infty$ and $\phi \leq 2 K$ on $\mathbf{R}^{d} \times[0, \infty)$.

\section{CONVERGENCE TO CODIMENSION $k$ MEAN CURVATURE FLOW}

In this section we consider examine asymptotic behavior of solutions of the generalized Ginzburg-Landau system in the case $d>k=p$.

For this purpose, it is convenient to introduce the normalized measure

$$
\nu_{t}^{\epsilon}=\frac{1}{\log (1 / \epsilon)} \mu_{t}^{\epsilon} .
$$


In the following, we assume that $\Gamma_{0}$ is a smooth embedded compact $(d-k)$-dimensional submanifold of $\mathbf{R}^{d}$, and that $\left\{\Gamma_{t}\right\}_{0<t \leq \tau}$ is a smooth codimension $k$ mean curvature flow starting from $\Gamma_{0}$, for some $\tau>0$. We let $\Gamma \subset \mathbf{R}^{d} \times[0, \tau]$ denote the set swept out by $\Gamma_{t}$, i.e.

$$
\Gamma=\bigcup_{0 \leq t \leq T} \Gamma_{t} \times\{t\}
$$

Also, we define

$$
\delta(x, t)=\operatorname{dist}\left(x, \Gamma_{+}\right)=\min _{y \in \Gamma_{+}}|x-y|
$$

Since $\Gamma$ is smooth and compact, we can find a number $\sigma_{0}>0$ and a smooth function $\eta$ such that

$$
\eta(x, l)= \begin{cases}\frac{1}{2} \delta^{2}(x, t) & \text { if } \delta(x, t) \leq \sigma_{0} \\ \sigma_{0}^{2} & \text { if } \delta(x, t) \geq 2 \sigma_{0}\end{cases}
$$

and

$$
\eta(x, t) \geq \frac{1}{2} \sigma_{0}^{2} \quad \text { whenever } \delta(x, t) \geq \sigma_{0} .
$$

Ambrosio and Soner [1] establish several properties of the function $\frac{1}{2} \delta^{2}$ in a recent paper. Their results immediately imply that $\eta$ has the following properties:

THEOREM 3.1. - For $(x, t)$ in a neighborhood of $\Gamma$, the matrix $\nabla^{2} \eta(x, t)$ has $k$ eigenvalues equal to one, and each of the remaining $d-k$ eigenvalues satisfies the estimate $\left|\lambda_{i}(x, t)\right| \leq C \delta(x, t)$. In particular, $\nabla^{2} \eta(x, t)$ is a projection onto a $k$-dimensional subspace when $(x, t) \in \Gamma$. Moreover,

$$
\nabla \eta_{t}-\Delta \nabla \eta=0 \quad \text { on } \Gamma \text {. }
$$

In fact, Ambrosio and Soner [1] show that for a smooth evolving manifold as above, $\nabla \eta_{t}(x, t)$ gives the normal velocity vector of $\Gamma_{t}$ at $(x, t) \in \Gamma$, and $\Delta \nabla \eta(x, t)$ equals the mean curvature vector. so the above equation precisely characterizes smooth codimension $k$ mean curvature flow.

We will use these results in the following form:

Corollary 3.1. - For any $\xi \in \mathbf{R}^{d}$ and all $(x, t)$ in a neighborhood of $\Gamma$ we have

$$
\nabla^{2} \eta \xi \cdot \xi \leq|\xi|^{2}
$$


Also, $\eta$ satisfies

$$
\eta_{t}-\Delta \eta \leq-k+C \eta
$$

in $\mathbf{R}^{d} \times[0, \tau]$.

Proof. - The first assertion is immediate from the above characterization of the eigenvalues of $\nabla^{2} \eta$ near $\Gamma$.

To verify the second assertion, first note that

$$
\eta_{t}=0, \quad \Delta \eta=k \quad \text { on } \Gamma .
$$

The first of these equalities holds because $\eta$ attains its minimum on $\Gamma$, and the second follows from the description of $\nabla^{2} \eta$ in Theorem 3.1. If we let $\phi=\eta_{t}-\Delta \eta$, we thus have (again using Theorem 3.1)

$$
\phi=-k, \quad \nabla \phi=0 \quad \text { on } \mathrm{\Gamma} \text {. }
$$

Given any $(x, t) \in \mathbf{R}^{d} \times[0, \tau]$, we can find $y \in \Gamma_{t}$ such that $|x-y|=\delta(x, t)$. We then have

$$
\begin{aligned}
\phi(x, t) & =\phi(y, t)+\nabla \phi(y, t) \cdot(x-y)+O\left(|x-y|^{2}\right) \\
& \leq-k+C \eta(x, t) .
\end{aligned}
$$

The following theorem is an easy consequence of these properties of $\eta$.

Theorem 3.2. - Suppose that $u^{\epsilon}: \mathbf{R}^{d} \rightarrow \mathbf{R}^{k}$ is a smooth solution of the generalized Ginzburg-Landau system (1.2) with $p=k$ and initial data $u^{\epsilon}(x, 0)=h^{\epsilon}(x)$ for which

$$
\int \eta d \nu_{0}^{\epsilon} \rightarrow 0
$$

as $\epsilon \rightarrow 0$. Then

$$
\int \eta d \nu_{t}^{\epsilon} \rightarrow 0
$$

Proof. - We use the smooth function $\eta$ defined above in the weighted energy estimate (2.3). Dropping a negative term and using (3.2) and the definition of $\mu_{t}^{\epsilon}$, we have

$$
\begin{aligned}
\frac{d}{d t} \int \eta d \mu_{t}^{\epsilon} & \leq \int\left(\eta_{t}-\Delta \eta\right) d \mu_{t}^{\epsilon}+\int\left(e_{\epsilon}\right)^{\frac{k-2}{2}} \nabla^{2} \eta \nabla u^{\epsilon} \cdot \nabla u^{\epsilon} d x \\
& \leq C \int \eta d \mu_{t}^{\epsilon}+\int-2\left(e_{\epsilon}\right)^{\frac{k}{2}}+\left(e_{\epsilon}\right)^{\frac{k-2}{2}} \nabla^{2} \eta \nabla u^{\epsilon} \cdot \nabla u^{\epsilon} d x
\end{aligned}
$$

Vol. 16, n 4-1999. 
Select $s \in\left(0, \sigma_{0}\right]$ such that $(3.1)$ holds on the set $\Gamma_{t}^{s} \equiv\{x \mid \delta(x, t)<s\}$. This number $s$ may be chosen uniformly for $t \in[0, \tau]$, so we may assume that $\left\|\nabla^{2} \eta\right\| / \eta \leq C$ on $\mathbf{R}^{d} \backslash \Gamma_{t}^{s}$ for some constant $C$, uniformly in $[0, \tau]$. Then

$$
\begin{aligned}
\int_{\Gamma_{i}^{s}}-2\left(e_{\epsilon}\right)^{\frac{k}{2}}+\left(e_{\epsilon}\right)^{\frac{k-2}{2}} \nabla^{2} \eta \nabla u^{\epsilon} \cdot \nabla u^{\epsilon} d x & \leq \int_{\Gamma_{i}^{s}}\left(e_{\epsilon}\right)^{\frac{k-2}{2}}\left(-2 e_{\epsilon}+\left|\nabla u^{\epsilon}\right|^{2}\right) d x \\
& \leq 0 .
\end{aligned}
$$

Moreover,

$$
\begin{aligned}
\int_{\mathbf{R}^{d} \backslash \Gamma_{t}^{*}}-2\left(e_{e}\right)^{\frac{k}{2}}+\left(e_{\epsilon}\right)^{\frac{k-2}{2}} \nabla^{2} \eta \nabla u^{\epsilon} \cdot \nabla u^{\epsilon} d x & \leq C \int_{\mathbf{R}^{d} \backslash \Gamma_{t}^{s}}\left(e_{\epsilon}\right)^{\frac{k-2}{2}} \eta\left|\nabla u^{\epsilon}\right|^{2} d x \\
& \leq C \int \eta d \mu_{t}^{\epsilon}
\end{aligned}
$$

The three preceding inequalities together yield

$$
\frac{d}{d t} \int \eta d \mu_{t}^{\epsilon} \leq C \int \eta d \mu_{t}^{\epsilon} .
$$

Gronwall's inequality now immediately implies that

$$
\int \eta d \mu_{t}^{\epsilon} \leq C \int \eta d \mu_{0}^{\epsilon}
$$

for all $0<t \leq \tau$. Dividing by $\log \frac{1}{\epsilon}$, we obtain the conclusion of the theorem.

Remark. - This proof may be seen as a Pohozaev-type estimate, as used for example in Bauman, Chen, Phillips, and Sternberg [3].

The hypotheses of the above theorem mention only the initial distribution of energy. If we assume in addition that $h^{\epsilon}$ exhibits a vortex-like structure along cross-sections of $\Gamma_{0}$, so that $\Gamma_{0}$ is a "topological defect", then we can strenthen the above result.

Because $\Gamma_{t}$ is assumed to be a smooth codimension $k$ manifold, at each $y \in \Gamma_{t}$ we may find vectors $n^{1}(y, t), \ldots, n^{k}(y, t) \in \mathbf{R}^{d}$ such that each $n^{\alpha}$ is normal to $\Gamma_{t}$, and $n^{\alpha} \cdot n^{\beta}=\delta_{\alpha \beta}$. We assume moreover that $\Gamma_{t}$ is orientable, so that $(y, t) \mapsto n^{\alpha}(y, t)$ may be taken to be smooth and globally well-defined on $\Gamma$.

For $y \in \Gamma_{t}$, we define $w^{\epsilon}(\cdot ; y, t): \mathbf{R}^{k} \rightarrow \mathbf{R}^{k}$ by

$$
w^{\epsilon}(x ; y, t):=u^{\epsilon}\left(y+x_{\alpha} n^{\alpha}(y, t), t\right)
$$


TheOREM 3.3. - Suppose that $u^{\epsilon}$ is a smooth solution of (1.2) with $p=k$ and initial data $u^{\epsilon}(x, 0)=h^{\epsilon}(x)$ satisfying

$$
\int_{\{\delta>\sigma\}} \frac{2}{k} e_{\epsilon}\left(h^{\epsilon}\right)^{k / 2} \leq C(\sigma)
$$

uniformly for $\epsilon>0$. If in addition there exists $\kappa, \sigma_{1}>0$ such that $\left\|\nabla u^{\epsilon}\right\|_{\infty} \leq \kappa / \epsilon$ and

$$
\lim _{\epsilon \rightarrow 0} \operatorname{deg}\left(w^{\epsilon}(\cdot ; y, 0) ; \partial B_{\sigma}\right)=1
$$

for all $y \in \Gamma_{0}, \sigma \in\left(0, \sigma_{1}\right]$, then

$$
\liminf _{\epsilon \rightarrow 0} \int \phi d \nu_{t}^{\epsilon} \geq K(k) \int_{\Gamma_{t}} \phi d H^{d-k}
$$

for all $\phi \in C_{0}^{\infty}\left(\mathbf{R}^{d}\right)$.

The constant $K(k)$ comes from Lemma 3.2 below.

Remark. - Theorems 3.2 and 3.3 taken together imply that if $\nu_{t}$ is any weak limit of $\nu_{t}^{\epsilon}$, then the support of $\nu_{t}$ exactly equals $\Gamma_{t}$.

As the sign of $\operatorname{deg}\left(w^{\epsilon}(\cdot ; y, t)\right)$ depends of the choice of $n^{1}, \ldots, n^{k}$, we may take it to be positive, without any loss of generality.

We assume $\sigma>0$ is fixed and we introduce the notation

$$
\begin{gathered}
K:=[0,1]^{d-k} \\
U:=B_{4 \sigma}^{k} \times K \subset \mathbf{R}^{d} .
\end{gathered}
$$

We denote typical points in $B_{4 \sigma}$ and $K$ as $x$ and $y$ respectively. Given a function $v^{\epsilon}: U \rightarrow \mathbf{R}^{k}$, we further define

$$
\begin{gathered}
m^{\epsilon}(y, t):=\operatorname{leb}^{1}\left(\left\{r \in[\sigma, 2 \sigma] \mid \operatorname{deg}\left(v^{\epsilon}(\cdot, y, t) ; \partial B_{r}\right)=1\right\}\right) \\
V_{t}^{\epsilon}:=\left\{y \in K \mid m^{\epsilon}(y, t)>\sigma / 2\right\}
\end{gathered}
$$

Here leb ${ }^{1}$ denotes 1-dimensional lebesgue measure. We may think of $V_{t}^{c}$ as the subset of points in $K$ at which the cross-section at time $t$ exhibits an isolated vortex, in a weak sense.

The following two estimates are proven in Jerrard [13] as Theorem 6.1 and Theorem 4.2 respectively. 
LeMma 3.1. - Let $v^{\epsilon} \in C\left([0, T] ; W^{1, \infty}\left(U ; \mathbf{R}^{k}\right)\right)$ with

$$
\left\|D v^{\epsilon}\right\|_{\infty} \leq \frac{\kappa}{\epsilon}
$$

for some $\kappa>0$, and assume that

$$
\operatorname{deg}\left(v^{\epsilon}(\cdot, y, 0) ; \partial B_{r}\right)=1
$$

for all $y \in K, r \in[\sigma, 4 \sigma]$, and that

$$
\int_{\{\sigma \leq|x| \leq 4 \sigma\}} \int_{K} \frac{2}{k} e_{\epsilon}\left(v^{\epsilon}\right)^{k / 2} d y d x \leq \kappa
$$

for all $t \in[0, T]$. Then

$$
\lim _{\epsilon \rightarrow 0} H^{d-k}\left(V_{t}^{\epsilon}\right)=H^{d-k}(K)=1,
$$

for all $t \in[0, T]$.

I.fmмa 3.2. - Suppose that $\phi^{\epsilon} \in W^{1, \infty}\left(\mathbf{R}^{k} ; \mathbf{R}^{k}\right)$ and that

$$
\left\|D \phi^{\epsilon}\right\|_{\infty} \leq \kappa / \epsilon
$$

and that

$$
\operatorname{leb} b^{1}\left(\left\{r \in[\sigma, 2 \sigma] \mid \operatorname{deg}\left(\phi^{\epsilon}, \partial B_{r}\right)=1\right\}\right) \geq \sigma / 2
$$

Then

$$
\int_{B_{2 \sigma}} \frac{2}{k} e_{\epsilon}\left(\phi^{\epsilon}\right)^{k / 2} d x \geq K(k) \ln (\sigma / \epsilon)-C(\kappa, k) .
$$

The constant $K(k)$ is given explicitly in Jerrard [13].

Using these we present the

Proof of Theorem 3.3.

1. For $\sigma \in\left(0, \sigma_{1}\right]$ to be determined, define $U$ as above.

First we define a map $\psi \in C^{\infty}(K \times[0, T] ; \Gamma)$ such that for every $t \in[0, T], \psi(\cdot, t)$ is a diffeomorphism of $K$ onto a subset of $\Gamma_{t}$. Now we define $\Psi: U \times[0, T] \rightarrow \mathbf{R}^{d}$ by

$$
\Psi(x, y, t):=\psi(y, t)+x_{\alpha} n^{\alpha}(\psi(y, t)) .
$$

Note that for fixed $(y, t), \Psi(\cdot, y, t)$ maps $\mathbf{R}^{k}$ onto the normal space to $\Gamma_{t}$ at $\psi(y, t)$. 
We may assume that $|\nabla \Psi| \leq C$ and that the $J \Psi \geq C^{-1}>0$, where $J \Psi=\operatorname{det} \nabla \Psi$ is the Jacobian of $\Psi$.

We also assume that $\sigma$ is small enough that $\Psi$ is one-to-one.

Finally we define $v^{\epsilon}(x, y, t):=u^{\epsilon}(\Psi(x, y, t), t)$.

2. We now verify that $v^{\epsilon}$ satisfies the hypotheses of Lemma 3.1 Since $u^{\epsilon}$ is assumed smooth, it is evident that the map $t \mapsto v^{\epsilon}(\cdot, \cdot, t)$ is continuous in the norm of $W^{1, \infty}$. We next compute

$$
\left|\nabla v^{\epsilon}\right| \leq\left|\nabla u^{\epsilon}\right||\nabla \Psi| \leq C\left|\nabla u^{\epsilon}\right| \leq C / \epsilon .
$$

Also, it is clear that

$$
v^{\epsilon}(x, y, t)=w^{\epsilon}(x ; \psi(y, t), t)
$$

so the condition on the degree of $v^{\epsilon}(\cdot, y, 0)$ follows immediately from (3.5) and our choice of $\sigma$.

Finally, note that $e_{\epsilon}\left(v^{\epsilon}\right)(x, y, t) \leq C e_{\epsilon}\left(u^{\epsilon}\right)(\Psi(x, y, t), t)$, and so

$$
\begin{aligned}
& \int_{\{\sigma \leq|x| \leq 4 \sigma\}} \int_{K} \frac{2}{k} e_{\epsilon}\left(v^{\epsilon}\right)^{k / 2}(x, y, t) d y d x \\
& \quad \leq \frac{C}{\min _{U} J \Psi} \int_{\{\sigma \leq|x| \leq 4 \sigma\}} \int_{K} \frac{2}{k} e_{\epsilon}\left(u^{\epsilon}\right)^{k / 2}(\Psi(x, y, t), t) J \Psi(x, y, t) d y d x \\
& \quad \leq C \int_{\{\delta(\cdot, t) \geq \sigma\}} \frac{2}{k} e_{\epsilon}\left(u^{\epsilon}\right)^{k / 2} \\
& \quad \leq C .
\end{aligned}
$$

The final equality follows from (3.4) by the calculation in the proof of Theorem 3.2.

3. Lemma 3.1 therefore asserts that (3.8) holds.

We now define, for $y \in \Gamma_{t}$

$$
\begin{gathered}
\tilde{m}^{\epsilon}(y, t):=\operatorname{leb}^{1}\left(\left\{r \in[\sigma, 2 \sigma] \mid \operatorname{deg}\left(w^{\epsilon}(\cdot ; y, t) ; \partial B_{r}^{k}\right)=1\right\}\right), \\
\tilde{V}_{t}^{\epsilon}:=\left\{y \in \Gamma_{t} \mid \tilde{m}^{\epsilon}(y, t)>\sigma / 2\right\} .
\end{gathered}
$$

It is clear that we can find a finite collection of maps $\left\{\Psi_{i}\right\}_{i=1}^{M}$ of the form described above such that

$$
\Gamma_{t} \subset \bigcup_{i=1}^{M} \Psi_{i}(U, t) .
$$


Thus (3.8) and (3.9) imply that

$$
\lim _{\epsilon \rightarrow 0} H^{d-k}\left(\Gamma_{t} \backslash \tilde{V}_{t}^{\epsilon}\right)=0
$$

4. For $x$ sufficiently close to $\Gamma_{t}$, let $p(x) \in \Gamma_{t}$ be the unique point of $\Gamma_{t}$ satisfying

$$
\delta(x, t)=|x-p(x)| .
$$

Fix $\sigma$ so small that $p(x)$ is well-defined on $\{\delta(\cdot, t) \leq 4 \sigma\}$. Note that for $y \in \tilde{V}_{t}^{\epsilon}$,

$$
\int_{\{\delta(\cdot, t) \leq 2 \sigma\} \cap p^{-1}(y)} \frac{2}{k|\log \epsilon|} e_{\epsilon}^{k / 2}(x) d H^{k}(x) \geq K(k)-\frac{C}{|\log \epsilon|} .
$$

This is an immediate consequence of Lemma 3.2 .

In the calculations below, $J p$ denotes the Jacobian of $p, J p:=$ $\left[\operatorname{det} d p d p^{T}\right]^{1 / 2}$. Here $d p$ denotes the gradient of $p$ considered as a map from $T_{x} \mathbf{R}^{d} \cong \mathbf{R}^{d}$ into $T_{p(x)} \Gamma_{t} \cong \mathbf{R}^{d-k}$, and thus is expressed as a $(d-k) \times d$ matrix, after choosing bases for the respective tangent spaces. In particular, with this definition the change of variables that we employ below is valid.

For every smooth, compactly supported $\phi$ we have

$$
\begin{aligned}
\int \phi d \nu_{t}^{\epsilon} & \geq \int_{\{\delta(\cdot, t) \leq 2 \sigma\}} \phi d \nu_{t}^{\epsilon} \\
& =I_{1}+I_{2}
\end{aligned}
$$

where

$$
I_{1}=\int_{\{\delta(\cdot, t) \leq 2 \sigma\}}[\phi(x)-J p(x) \phi(p(x))] d \nu_{t}^{\epsilon}
$$

and, by a version of the co-area formula,

$$
\begin{aligned}
I_{2} & =\int_{\{\delta(, t) \leq 2 \sigma\}} J p(x) \phi(p(x)) \frac{2}{k} e_{\epsilon}^{k / 2}(x) \frac{d x}{|\log \epsilon|} \\
& =\int_{\Gamma_{t}} \phi(y)\left[\int_{\{\delta(, t) \leq 2 \sigma\} \cap p^{-1}(y)} \frac{2}{k|\log \epsilon|} e_{\epsilon}^{k / 2}(x) d H^{k}(x)\right] d H^{d-k}(y) \\
& \geq \int_{\tilde{V}_{t}^{e}} \phi(y)\left[K(k)-\frac{C}{|\log \epsilon|}\right] d H^{d-k}(y)
\end{aligned}
$$

In the last step we have used (3.11). 
5. Fix any subsequence and a measure $\nu$ such that $\nu_{t}^{\epsilon_{n}} \rightarrow \nu$. By Theorem 3.2, we know that spt $\nu \subset \Gamma_{t}$. It follows that

$$
I_{1} \rightarrow \int_{\Gamma_{t}}[\phi(y)-J p(y) \phi(y)] d \nu
$$

We will show in Lemma 3.3 below that $J p(y)-1$ for $y \in \Gamma_{t}$. Thus $I_{1}$ vanishes as $\epsilon \rightarrow 0$.

Also, (3.12) and (3.10) evidently imply that

$$
\liminf _{\epsilon \rightarrow 0} I_{2} \geq K(k) \int_{\Gamma_{t}} \phi(y) d H^{d-k}(y) .
$$

Lemma 3.3. - For $p$ as defined above and $y \in \Gamma_{t}, J p(y)=1$.

Proof. - Fix $y \in \Gamma_{t}$ and orthonormal vectors $\tau_{1}, \ldots, \tau_{d-k}$ which span $T_{y} \Gamma_{t}$. Taking the standard basis $e_{1}, \ldots, e_{d}$ as a basis for $T_{y} \mathbf{R}^{d}$, the matrix $d p$ has the form

$$
(d p)_{i j}=\tau_{i} \cdot \nabla p^{j}, \quad i=1, \ldots, d-k, \quad j=1, \ldots, d .
$$

After a rclabelling we may assume that $e_{i}=\tau_{i}$ for $i=1, \ldots, d \quad k$ and that $e_{d-k+1}, \ldots, e_{d}$ are normal to $\Gamma_{t}$ at $\mathrm{y}$.

We claim that

$$
(d p)_{i j}(y)=\delta_{i j} \quad \text { for } i=1, \ldots, d-k .
$$

Indeed, for any $i=1, \ldots, d-k$, by the definition of $p$,

$$
\begin{aligned}
\left|p\left(y+h e_{i}\right)-y-h e_{i}\right| & =\delta\left(y+h e_{i}\right) \\
& =\delta(y)+h e_{i} \cdot \nabla \delta+O\left(h^{2}\right) \\
& =O\left(h^{2}\right)
\end{aligned}
$$

since $\nabla \delta$ is normal to $\Gamma_{t}$. Since $p(y)=y$, this implies that

$$
\left|\frac{p\left(y+h e_{i}\right)-p(y)}{h}-e_{i}\right|=O(h),
$$

which implies (3.13).

Also, for $j>d-k$ and $h$ sufficiently small, similar reasoning shows that $p\left(y+h e_{j}\right)=p(y)$. Thus $(d p)_{i j}=0$ whenever $j>d-k$. With (3.13) and the definition of $J p$, this implies the conclusion of the lemma. 
In the remainder of this section, we briefly indicate a way to construct initial data $h^{\epsilon}$ for (1.2) in such a way that the resulting solutions, if smooth, will satisfy the hypotheses of Theorems 3.2 and 3.3 .

We impose some topological restrictions on $\Gamma_{0}$ by assuming that there exist smooth, bounded, open sets $\mathcal{O}_{\alpha}, \alpha=1, \ldots, k$, such that

$$
\Gamma_{0}=\bigcap_{\alpha=1}^{h} \partial \mathcal{O}_{\alpha}
$$

For $\alpha=1, \ldots, k$, let $\tilde{d}^{\alpha}$ be the (signed) distance to $\partial \mathcal{O}_{\alpha}$, so that

$$
\tilde{d}^{\alpha}(x)= \begin{cases}-\operatorname{dist}\left(x, \partial \mathcal{O}^{\alpha}\right) & \text { if } x \in \mathcal{O}_{\alpha} \\ \operatorname{dist}\left(x, \partial \mathcal{O}^{\alpha}\right) & \text { if } x \in \mathbf{R}^{d} \backslash \mathcal{O}_{\alpha}\end{cases}
$$

Since the sets $\mathcal{O}_{\alpha}$ are assumed smooth, each function $\tilde{d}^{\alpha}$ is smooth near $\partial \mathcal{O}_{\alpha}$. We assume in addition to (3.14) that

$$
\nabla \tilde{d}^{\alpha} \cdot \nabla \tilde{d}^{\beta}=\delta_{\alpha i 3}
$$

on $\Gamma_{0}$.

For $\alpha=1, \ldots, k$, let $d^{\alpha}$ be smooth functions such that

$$
\begin{gathered}
d^{\alpha}=\tilde{d}^{\alpha} \quad \text { in an neighborhood of } \Gamma_{0}, \\
\operatorname{sgn}\left(\tilde{d}^{\alpha}\right)=\operatorname{sgn}\left(d^{\alpha}\right), \quad \frac{1}{2}\left|\tilde{d}^{\alpha}\right| \leq\left|d^{\alpha}\right| \leq 2\left|\tilde{d}^{\alpha}\right| \quad \text { on } \mathbf{R}^{d} .
\end{gathered}
$$

Let $d: \mathbf{R}^{d} \rightarrow \mathbf{R}^{k}$ be the vector-valued function whose $\alpha$ th component is $d^{\alpha}$. Note that $d$ is related to the ordinary distance function $\delta(\cdot, 0)$, defined above, by

$$
C^{-1} \delta(x, 0) \leq|d(x)|<C \delta(x, 0)
$$

Finally, note that assumption (3.14) implies that $\tilde{d} /|\tilde{d}| \rightarrow k^{-1 / 2}(1, \ldots, 1)$ as $|x| \rightarrow \infty$, so we may find $d$ satisfying the above conditions, for which there exists some number $M$ such that

$$
d /|d|=k^{-1 / 2}(1, \ldots, 1) \quad \text { for all }|x|>M .
$$

Remark. - 1. Assumption (3.14) appears to be a necessary condition for the existence of initial data with the required properties. Given (3.17), one can modify the sets $\mathcal{O}_{\alpha}$ locally near $\Gamma_{0}$ to arrange that (3.15) be satisfied. 
2. Assumption (3.14) is satisfied by any $\Gamma_{0}$ which can be embedded as a codimension 1 manifold in $\mathbf{R}^{d-k+1}$. Also, it is clearly preserved under homotopy.

Let $v: \mathbf{R}^{k} \rightarrow \mathbf{R}^{k}$ be a function of the form $v(x)=\rho(|x|) \frac{x}{|x|}$, for a scalar function $\rho$ such that

$$
\rho(0)=\rho^{\prime}(0)=0, \quad 1-C e^{-|x| / C} \leq \rho(|x|)<1, \quad \rho^{\prime}(|x|) \leq C e^{-C /|x|} .
$$

Then for $e(v)=\frac{1}{2}|D v|^{2}+W(v)$, we have

$$
e(v)(x)=\frac{k-1}{|x|^{2}}+O\left(e^{-C /|x|}\right)
$$

as $|x| \rightarrow \infty$.

We define

$$
h^{\epsilon}(x)=v\left(\frac{d(x)}{\epsilon}\right) .
$$

One can then verify that

$$
\left.\nu_{0}^{\epsilon} \rightarrow K(k) H^{d-k}\right|_{\Gamma_{0}}
$$

in the sense of distributions, and it is clear that (3.5) holds. Moreover, one can verify that $h^{\epsilon}$ satisfies the hypotheses of Proposition 2.2, and thus that a smooth solution $u^{\epsilon}$ with $h^{\epsilon}$ as initial data satisfies

$$
\left\|\nabla u^{\epsilon}\right\|_{\infty} \leq C / \epsilon
$$

So $h^{\epsilon}$ has all of the desired properties.

\section{SMALL ENERGY REGULARITY}

In this section we establish a small energy regularity theorem for solutions of the generalized Ginzburg-Landau system. The basic argument we follow was introduced by Schoen [22] for stationary harmonic maps and generalized by Struwe [24] and Chen and Struwe [8] to the case of heat flow for harmonic maps and for Ginzburg-Landau type approximations of harmonic maps.

The proof relies on a monotonicity lemma and a Bochner-type inequality, that is, a differential inequality which is satisfied by the energy. The main 
novelty here is the observation that these estimates are available in this more general context, as well as the fact that our result is local in nature. In problems involving asymptotic behavior of solutions of Ginzburg-Landau type systems, global energy estimates, independent of $\epsilon$, typically do not hold. Thus the local character of our estimates is very useful in these applications.

Small energy regularity results of the sort that we establish here can be used with covering arguments to deduce partial regularity results, as in Chen and Struwe [8].

We start by establishing a monotonicity formula, which we get by putting an appropriate test function $\eta$ in the identity (2.5). We first define this function:

Let $\gamma:[0, \infty) \rightarrow[0,1]$ be a smooth nonincreasing function such that

$$
\gamma(r)= \begin{cases}1 & \text { if } r \leq 1 / 4 \\ 0 & \text { if } r \geq 1 / 2\end{cases}
$$

Also, define $\rho: \mathbf{R}^{d} \times(0, \infty) \rightarrow \mathbf{R}$ by

$$
\rho(x, t)=(4 \pi t)^{-\frac{d-p}{2}} \exp \left(\frac{-|x|^{2}}{4 t}\right) .
$$

We fix $\left(x_{0}, t_{0}\right)$ and let $\eta(x, t)=\gamma\left(\left|x_{0}-x\right|\right) \rho\left(x_{0}-x, t_{0}-t\right)$, for $x \in \mathbf{R}^{d}, t<t_{0}$. In what follows we will for simplicity take $x_{0}=0$ and write $\tau$ for $t_{0}-t$. We have

$$
\begin{gathered}
\eta_{t}=\left(\frac{d-p}{2 \tau}-\frac{|x|^{2}}{4 \tau^{2}}\right) \eta, \quad \nabla \eta=\left(\frac{\nabla \gamma}{\gamma}-\frac{x}{2 \tau}\right) \eta, \\
\nabla^{2} \eta=\left[\frac{\nabla^{2} \gamma}{\gamma}-\frac{\nabla \gamma}{\gamma} \otimes \frac{x}{2 \tau}-\frac{x}{2 \tau} \otimes \frac{\nabla \gamma}{\gamma}+\frac{x \otimes x}{4 \tau^{2}}-\frac{I}{2 \tau}\right] \eta,
\end{gathered}
$$

where $I$ is the identity matrix. We then have

$$
\begin{gathered}
\eta_{t}+\Delta \eta=\left(\Delta \gamma-\frac{\nabla \gamma \cdot x}{\tau}\right) \frac{\eta}{\gamma}-\frac{p}{2 \tau} \eta \\
\nabla^{2} \eta \nabla u^{\epsilon} \cdot \nabla u^{\epsilon}=\left(\nabla^{2} \gamma \nabla u^{\epsilon} \cdot \nabla u^{\epsilon}-\left(\nabla \gamma \cdot \nabla u^{\epsilon}\right)\left(\frac{x}{\tau} \cdot \nabla u^{\epsilon}\right)\right) \frac{\eta}{\gamma} \\
+\left(\frac{\left|x \cdot \nabla u^{\epsilon}\right|^{2}}{4 \tau^{2}}-\frac{\left|\nabla u^{\epsilon}\right|^{2}}{2 \tau}\right) \eta
\end{gathered}
$$




$$
\frac{\left|\nabla \eta \cdot \nabla u^{\epsilon}\right|^{2}}{\eta}=\left(\frac{\left|\nabla \gamma \cdot \nabla u^{\epsilon}\right|^{2}}{\gamma}-\left(\nabla \gamma \cdot \nabla u^{\epsilon}\right)\left(\frac{x}{\tau} \cdot \nabla u^{\epsilon}\right)\right) \frac{\eta}{\gamma}+\frac{\left|x \cdot \nabla u^{\epsilon}\right|^{2}}{4 \tau^{2}} \eta
$$

So with this choice of $\eta$ in (2.4) we obtain (using the fact that $\eta$ is nonnegative)

$$
\begin{aligned}
\frac{\partial}{\partial t} \int \eta d \mu_{t}^{\epsilon} \leq \int\left(\Delta \gamma-\nabla \gamma \cdot \frac{x}{\tau}\right) \frac{\eta}{\gamma} d \mu_{t}^{\epsilon}+\int\left(\left(e_{\epsilon}\right)^{\frac{p-2}{2}} \frac{\left|\nabla u^{\epsilon}\right|^{2}}{2 \tau}-\frac{1}{\tau}\left(e_{\epsilon}\right)^{p / 2}\right) \eta d x \\
+\int\left(e_{\epsilon}\right)^{\frac{p-2}{2}}\left(\frac{\left|\nabla u^{\epsilon} \cdot \nabla \gamma\right|^{2}}{\gamma^{2}}+\frac{\nabla^{2} \gamma}{\gamma} \nabla u^{\epsilon} \cdot \nabla u^{\epsilon}\right) \eta d x \\
\leq \int\left(\Delta \gamma-\nabla \gamma \cdot \frac{x}{\tau}\right) \frac{\eta}{\gamma} d \mu_{t}^{\epsilon} \\
+\int\left(e_{\epsilon}\right)^{\frac{p-2}{2}}\left(\frac{\left|\nabla u^{\epsilon} \cdot \nabla \gamma\right|^{2}}{\gamma^{2}}+\frac{\nabla^{2} \gamma}{\gamma} \nabla u^{\epsilon} \cdot \nabla u^{\epsilon}\right) \eta d x
\end{aligned}
$$

By the definition of $\gamma$, the integrals on the right-hand side above are supported on $\{x: 1 / 4 \leq|x| \leq 1 / 2\}$. Recalling that $|\nabla \gamma|^{2} / \gamma \leq\|\gamma\|_{C^{2}}$, we have

$$
\frac{\partial}{\partial t} \int \eta d \mu_{t}^{\epsilon} \leq C \int_{\{1 / 4 \leq|x| \leq 1 / 2\}}\|\gamma\|_{C^{2}}\left(1+\frac{|x|}{\tau}\right) \frac{\eta}{\gamma} d \mu_{t}^{\epsilon}
$$

Also, for $|x| \geq 1 / 4$,

$$
\left(1+\frac{|x|}{\tau}\right) \frac{\eta}{\gamma}=\left(1+\frac{|x|}{\tau}\right)(4 \pi \tau)^{-\frac{d-p}{2}} \exp \left(-\frac{|x|^{2}}{4 \tau}\right) \leq C(d-p) .
$$

Thus we have established the following local monotonicity formula.

LEMMA 4.1. - The measures $\mu_{t}^{\epsilon}$ satisfy the estimate

$$
\frac{\partial}{\partial t} \int \eta d \mu_{t}^{\epsilon} \leq C(\gamma, d-p) \mu_{t}^{\epsilon}\left(B_{1 / 2}\right)
$$

Before stating our small-energy regularity result, we introduce some notation. For $x_{0} \in \mathbf{R}^{d}, r>0$, and $0 \leq t<t_{0}$, let

$$
\alpha_{r}^{\epsilon}\left(t ; x_{0}, t_{0}\right):=\int \gamma\left(\frac{\left|x_{0}-x\right|}{r}\right) \rho\left(x_{o}-x, t_{0}-t\right) d \mu_{t}^{\epsilon}(x),
$$

where $\gamma$ and $\rho$ are defined at the beginning of this section: We write $\alpha^{\epsilon}$ to mean $\alpha_{1}^{\epsilon}$. Note that the $\alpha_{r}^{\epsilon}$ is scale-invariant in the following Vol. $16, \mathrm{n}^{\circ} 4-1999$. 
sense: Given a function $u^{\epsilon}$ solving (1.2), we may define a rescaled function by $\tilde{u}(x, t)=u^{\epsilon}\left(x_{0}+R x, R^{2} t\right)$. We also define $\tilde{\epsilon}=\epsilon / R$, $\tilde{E}(x, t)=2 / p\left(e^{\tilde{\epsilon}}(\tilde{u})\right)^{p / 2}$. As remarked in the introduction, $\tilde{u}$ solves the system (1.2) with scaling $\tilde{\epsilon}$, and

$$
\tilde{E}(x, t)=R^{p} E^{\epsilon}\left(x_{0}+R x, R^{2} t\right)
$$

Thus, using the fact that $\rho(R y, s)=R^{d-p} \rho\left(y, s / R^{2}\right)$ we obtain by a change of variables

$$
\begin{aligned}
\alpha_{r}^{\epsilon}\left(t ; x_{0}, t_{0}\right) & -\int \gamma\left(\frac{\left|x_{0}-x\right|}{r}\right) \rho\left(x_{0}-x, t_{0}-t\right) E^{\epsilon}(x, t) d x \\
& =\int \gamma\left(\frac{R|x|}{r}\right) \rho\left(x, \frac{t-t_{0}}{R^{2}}\right) \tilde{E}\left(x, \frac{t}{R^{2}}\right) d x \\
& =\tilde{\alpha}_{r / R}^{\tilde{\epsilon}}\left(\frac{t}{R^{2}} ; x, \frac{t_{0}}{R^{2}}\right)
\end{aligned}
$$

In particular, by taking $R=r$ we can convert statements about $\alpha_{r}^{\epsilon}$ to statements about $\alpha^{\epsilon}$.

We now change notation, using $\eta$ to denote a small constant which will be chosen below. We also introduce the notation

$$
\mathcal{P}_{s}\left(x_{0}, t_{0}\right):=\left\{(x, t)\left|t \in\left[t_{0}-s^{2}, t\right],\right| x-x_{0} \mid \leq s\right\}
$$

We now have

THEOREM 4.1 (local small-energy regularity). - Suppose that $u^{\epsilon}$ is a solution of (1.2) on $B_{r} \times\left[T_{0}, T_{1}\right]$, with $\epsilon \leq r$. Suppose also that there exists $\kappa>0$ such that for all $x \in B_{r}, s \leq r$ with $B_{s}(x) \subset B_{r}$, and all $t \in\left[T_{0}, T_{1}\right]$ we have

$$
\mu_{t}^{\epsilon}\left(B_{s}(x)\right) \leq \kappa s^{d-p}
$$

Then there are positive constants $\eta, \rho_{0}$, and $C$ such that if

$$
\alpha_{r}^{\epsilon}\left(t_{0}-\tau^{2} ; x_{0}, t_{0}\right) \leq \eta
$$

for some $\left(x_{0}, t_{0}\right) \in B_{r / 4} \times\left[T_{0}+\tau^{2}, T_{1}\right]$ and $\tau \in(0, r \sqrt{\eta}]$, then we have

$$
E^{\epsilon}(x, t) \leq \frac{C}{\left(\rho_{0} \tau\right)^{p}} \quad \text { in } \mathcal{P}_{\rho_{0} \tau}\left(x_{0}, t_{0}\right)
$$


Proof. -1 . We first claim that it suffices to establish the theorem under the assumption that $\tau=r \sqrt{\eta}$. Indeed, if $\tau^{2}<r^{2} \eta$ then we define $\tilde{r}$ by insisting that $\tau=\tilde{r} \sqrt{\eta}$. Note that $\tilde{r}<r$, and so $\gamma(|x| / \tilde{r}) \leq \gamma(|x|)$ for all $x$. Thus

$$
\alpha_{\tilde{r}}^{\epsilon}\left(t_{0}-\tau ; x_{0}, t_{0}\right) \leq \alpha_{r}^{\epsilon}\left(t_{0}-\tau ; x_{0}, t_{0}\right) \leq \eta
$$

Clearly also (4.2) continues to hold if $r$ is replaced by $\tilde{r}$. We may then use $\tilde{r}$ instead of $r$ in the proof, and the desired equality will be satisfied.

Next, by rescaling we may set $r=1$. Thus we assume that

$$
\alpha^{\epsilon}\left(t_{0}-\eta ; x_{0}, t_{0}\right) \leq \eta, \quad \tau^{2}=\eta
$$

The constant $\eta \in(0,1]$ will be fixed at the end of the proof. After thesc normalizations, (4.2) implies that

$$
\mu_{t}^{\epsilon}\left(B_{1}\right) \leq \kappa
$$

2. For $\rho_{0} \in(0,1 / 4]$ to be chosen, let

$$
f(\sigma)=\left(2 \rho_{0} \sqrt{\eta}-\sigma\right)^{p} \max _{\mathcal{P}_{\sigma}\left(x_{0}, t_{0}\right)} E(x, t)
$$

Take $\sigma_{0} \in\left(0,2 \rho_{0} \sqrt{\eta}\right)$ and $(\bar{x}, \bar{t}) \in \mathcal{P}_{\sigma_{0}}\left(x_{0}, t_{0}\right)$ such that

$$
f\left(\sigma_{0}\right)=\max _{\left[0,2 \rho_{0} \sqrt{\eta}\right]} f(\sigma), \quad E(\bar{x}, \bar{t})=\max _{\mathcal{P}_{\sigma_{0}}\left(x_{0}, t_{0}\right)} E(x, t)=\mathcal{E}
$$

Let $\bar{\sigma}=\rho_{0} \sqrt{\eta}-\sigma_{0} / 2$. Then

$$
\begin{aligned}
(2 \bar{\sigma})^{p} \mathcal{E}=f\left(\sigma_{0}\right) & \geq f\left(\sigma_{0}+\bar{\sigma}\right) \\
& =\bar{\sigma}^{p} \sup _{\mathcal{P}_{\sigma_{0}+\bar{\sigma}\left(x_{0}, t_{0}\right)} E} E \\
& \geq \bar{\sigma}^{p} \sup _{\mathcal{P}_{\bar{\sigma}}(\bar{x}, \bar{t})} E
\end{aligned}
$$

Thus $E(x, t) \leq 2^{p} \mathcal{E}$ in $\mathcal{P}_{\bar{\sigma}}(\bar{x}, \bar{t})$. Estimate (2.6) now implies that

$$
E_{t}-\Delta E-\frac{p-2}{2} \frac{\nabla^{2} E \nabla u^{\epsilon} \cdot \nabla u^{\epsilon}}{e_{\epsilon}} \leq C\left(2^{p} \mathcal{E}\right)^{2 / p} E=128 \mathcal{E}^{2 / p} E
$$

in $\mathcal{P}_{\bar{\sigma}}(\bar{x}, \bar{l})$, since $(2.6)$ holds with $C=32$.

Vol. $16, n^{\circ} 4-1999$. 
Note also that it now suffices to show that $\bar{\sigma}^{p} \mathcal{E} \leq C$ for appropriate choices of $\eta, \rho_{0}$. Indeed, if we have this estimate, then

$$
\begin{aligned}
\left(\rho_{0} \tau\right)^{p} \max _{\mathcal{P}_{\rho_{0} \tau}\left(x_{0}, t_{0}\right)} E(x, t) & =f\left(\rho_{0} \tau\right) \\
& \leq f\left(\sigma_{0}\right) \\
& =(2 \bar{\sigma})^{p} \mathcal{E} \\
& \leq C,
\end{aligned}
$$

which is the conclusion of the theorem.

3. Let $\tilde{\sigma}=\bar{\sigma} \wedge \mathcal{E}^{-1 / p}$ and

$$
w(x, t)=\exp \left(128 \mathcal{E}^{2 / p}(\bar{t}-t)\right) E(x, t) .
$$

Then

$$
w_{t}-\Delta w-\frac{p-2}{2} \frac{\nabla^{2} w \nabla u^{\epsilon} \cdot \nabla u^{\epsilon}}{e_{\epsilon}} \leq 0,
$$

and $w \leq e^{128} E$ in $\mathcal{P}_{\tilde{\sigma}}(\bar{x}, \bar{t})$. The coefficients in the above equation satisfy

$$
|\xi|^{2} \leq\left(\delta_{i j}+\frac{p-2}{2} \frac{u_{x_{i}}^{\epsilon} \cdot u_{x_{j}}^{\epsilon}}{e_{\epsilon}\left(u^{\epsilon}\right)}\right) \xi_{i} \xi_{j} \leq p|\xi|^{2},
$$

so by a parabolic Harnack inequality for nondivergence structure equations, see Krylov and Safonov [15], which depends only on the above bounds on the coefficients, we have

$$
\begin{aligned}
\mathcal{E}=w(\bar{x}, \bar{t}) & \leq C \tilde{\sigma}^{-(d+2)} \int_{\mathcal{P}_{\tilde{\sigma}}(\bar{x}, \bar{t})} w(x, t) d x d t \\
& \leq C \tilde{\sigma}^{-(d+2)} \int_{\mathcal{P}_{\tilde{\sigma}}(\bar{x}, \bar{t})} E(x, t) d x d t
\end{aligned}
$$

4. Since $\eta \leq 1$ and $\rho_{0} \leq 1 / 4$,

$$
\tilde{\sigma} \leq \bar{\sigma} \leq \rho_{0} \sqrt{\eta} \leq 1 / 4
$$

and so for $(x, t) \in \mathcal{P}_{\tilde{\sigma}}(\bar{x}, \bar{t})$, we have

$$
\gamma(|\bar{x}-x|) \rho\left(\bar{x}-x, \bar{t}+\tilde{\sigma}^{2}-t\right) \geq \tilde{\sigma}^{-(d-p)} \exp (-1 / 4)
$$

Thus

$$
\begin{aligned}
1 \wedge \bar{\sigma}^{p} \mathcal{E} & =\tilde{\sigma}^{p} \mathcal{E} \\
& \leq C \tilde{\sigma}^{-2} \int_{\mathcal{P}_{\bar{\sigma}}(\bar{x}, \bar{t})} \gamma(|\bar{x}-x|) \rho\left(\bar{x}-x, \bar{t}+\tilde{\sigma}^{2}-t\right) E(x, t) d x d t \\
& \leq C \tilde{\sigma}^{-2} \int_{\bar{t}-\tilde{\sigma}^{2}}^{\bar{t}} \alpha^{\epsilon}\left(t ; \bar{x}, \bar{t}+\tilde{\sigma}^{2}\right) d t \\
& \leq C \alpha^{\epsilon}\left(\iota_{0}-\eta ; \bar{x}, \bar{t}+\tilde{\sigma}^{2}\right)+C(\kappa)\left(\bar{t}-\left(t_{0}-\eta\right)\right)
\end{aligned}
$$

by the monotonicity formula, Lemma 4.1 . 
Rccall that by construction, $\bar{t}<t_{0}$, so the last term on the right hand side above is bounded by $C(\kappa) \eta$.

5. By translation, we may set $x_{0}=0$, and we define $\tilde{t}:=\bar{t}+\tilde{\sigma}^{2}-t_{0}$. Observe that by construction we have

$$
|\bar{x}| \leq 2 \rho_{0} \sqrt{\eta},|\tilde{t}| \leq 4 \rho_{0}^{2} \eta
$$

We now claim that if $\rho_{0}$ is sufficiently small, then

$$
\alpha^{\epsilon}\left(t_{0}-\eta ; \bar{x}, \bar{t}+\tilde{\sigma}^{2}\right) \leq 4 \alpha^{\epsilon}\left(t_{0}-\eta ; x_{0}, t_{0}\right)+\eta \leq 5 \eta .
$$

We write

$$
\alpha^{\epsilon}\left(t_{0}-\eta ; \bar{x}, \bar{t}+\tilde{\sigma}^{2}\right) \cdot 4 \alpha^{\epsilon}\left(t_{0}-\eta ; 0, t_{0}\right)=I_{1}+I_{2}+I_{3}
$$

where

$$
\begin{gathered}
I_{1}=\int[\gamma(|\bar{x}-y|)-\gamma(|y|)] \rho(\bar{x}-y, \eta+\tilde{t}) E\left(y, t_{0}-\eta\right) d y \\
I_{2}=\int \gamma(|y|)[\rho(\bar{x}-y, \eta+\tilde{t})-2 \rho(y, \eta+\tilde{t})] E\left(y, t_{0}-\eta\right) d y \\
I_{3}=2 \int \gamma(|y|)[\rho(y, \eta+\tilde{t})-2 \rho(y, \eta)] E\left(y, t_{0}-\eta\right) d y
\end{gathered}
$$

Recalling that $\gamma \equiv 1$ on $B_{1 / 4}(0)$, we have

$$
\begin{aligned}
I_{1} \leq & \|\nabla \gamma\|_{\infty}|\bar{x}| \\
& \left(\int_{B} E\left(y, t_{0}-\eta\right) d y\right) \\
& \quad \times \sup \{\rho(\bar{x}-y, t) \quad|| y|\geq 1 / 4,| \bar{x}-y \mid \geq 1 / 4, \quad t \geq 0\}
\end{aligned}
$$

if $1 / 8>2 \rho_{0} \sqrt{\eta} \geq|\bar{x}|$, which may be achieved, for given $\eta$, by adjusting $\rho_{0}$. Taking $\rho_{0}$ still smaller yields $I_{1} \leq \eta / 3$.

To estimate $I_{2}$, notc that if $\rho(\bar{x}-y, \eta+\tilde{t})-2 \rho(y, \eta+\tilde{t})>0$ then

$$
\exp \left(\frac{|y|^{2}-|\bar{x}-y|^{2}}{4(\eta+\hat{t})}\right) \geq 2
$$

We rewrite this inequality as

$$
2 \bar{x} \cdot(y-\bar{x}) \geq 4(\eta+\tilde{t})\left(\log 2-\frac{|\bar{x}|^{2}}{1(\eta+\tilde{t})}\right) .
$$

Vol. 16, n 4-1999. 
This implies that

$$
\frac{|\bar{x}-y|^{2}}{4(\eta+\tilde{t})} \geq \frac{\eta+\tilde{t}}{|\bar{x}|^{2}}\left(\log 2-C \rho_{0}\right)^{2} \geq C^{-1} \rho_{0}^{-2}
$$

using (4.6). Thus

$$
\begin{aligned}
\rho(\bar{x}-y, \eta+\tilde{t}) & \leq(\eta+\tilde{t})^{\frac{p-d}{2}} \exp \left(-C^{-1} \rho_{0}^{-2}\right) \\
& \leq \eta / 3 \kappa
\end{aligned}
$$

if $\rho_{0}$ is chosen small enough. With (4.3) this implies that $I_{2} \leq \eta / 3$.

The estimate of $I_{3}$ is very similar to that of $I_{2}$, so we omit it. Thus we have proven our claim.

6. Putting together steps 4 and 5 , we find that

$$
1 \wedge \bar{\sigma}^{p} \mathcal{E} \leq C \eta
$$

Taking $\eta$ small now gives

$$
\bar{\sigma}^{p} \mathcal{E} \leq C \eta
$$

As remarked in step 2, this immediately yields the conclusion of the theorem.

\section{SOME VARIATIONS}

By modifying the argument of the small energy regularity theorem, we obtain slightly different results which will be useful later on.

Proposition 5.1. - Suppose that $u^{\epsilon}$ solves (1.1) on $B_{r} \times\left[T_{0}, T_{1}\right]$, where $\epsilon<r$. Assume also that there exists $\kappa>0$ such that

$$
\mu_{t}^{\epsilon}\left(B_{s}(x)\right) \leq \kappa s^{d-2}
$$

for all $t \in\left[T_{0}, T_{1}\right]$ and all $B_{s}(x) \subset B_{r}$, and that

$$
E^{\epsilon}\left(x, T_{0}\right) \leq \kappa / r^{2} \quad \text { in } B_{r} .
$$

Then there exist constants $C(\kappa), \tau(\kappa)$ such that

$$
E^{\epsilon}(x, t) \leq C / r^{2} \quad \text { in } B_{r / 2} \times\left[T_{0}, T_{0}+r^{2} \tau\right]
$$

Remark. - Note that this applies only to the usual Ginzburg-Landau system with quadratic growth. 
Proof. - 1. As in the proof of Proposition 4.1, it suffices to prove the result for $r=1 \geq \epsilon$. We may also assume by a translation that $T_{0}=0$.

Take $x_{0} \in B_{1 / 2}, t_{0}<1 / 16$ to be fixed later, and define

$$
f(\sigma)=\left(\sqrt{t_{0}}-\sigma\right)^{2} \sup _{\mathcal{P}_{\sigma}\left(x_{0}, t_{0}\right)} E^{\epsilon}(x, t) .
$$

Exactly as in the proof of Proposition 4.1 we select $\sigma_{0} \in\left(0, \sqrt{t_{0}}\right)$ and $(\bar{x}, \bar{t}) \in \mathcal{P}_{\sigma_{0}}$ such that

$$
f\left(\sigma_{0}\right)=\max _{\left[0, \sqrt{t_{0}}\right]} f(\sigma) \quad E^{\epsilon}(\bar{x}, \bar{t})=\max _{\mathcal{P}_{\sigma_{0}}(\bar{x}, \bar{t})} E(x, t):=\mathcal{E} .
$$

We further define $\bar{\sigma}:=\left(\sqrt{t_{0}}-\sigma_{0}\right) / 2$. Following the argument of steps $1-4$ of the proof of Proposition 4.1, we find that

$$
1 \wedge \bar{\sigma}^{2} \mathcal{E} \leq C \tilde{\sigma}^{-2} \int_{\bar{t}-\tilde{\sigma}^{2}}^{\bar{t}} \alpha^{\epsilon}\left(t, \bar{x}, \bar{t}+\tilde{\sigma}^{2}\right) d t
$$

for some $\tilde{\sigma} \leq \bar{\sigma}$.

2. By the monotonicity lemma, the definition of $\alpha^{\epsilon}$ and the assumed $L^{\infty}$ bounds on $E^{\epsilon}(\cdot, 0)$,

$$
\begin{aligned}
\alpha^{\epsilon}\left(t ; \bar{x}, \bar{t}+\tilde{\sigma}^{2}\right) d t & \leq \alpha^{\epsilon}\left(0 ; \bar{x}, \bar{t}+\tilde{\sigma}^{2}\right)+C(\kappa) t \\
& \leq \kappa\left(\bar{t}+\tilde{\sigma}^{2}\right)+C(\kappa) t
\end{aligned}
$$

From the definitions we have $\bar{t}+\tilde{\sigma}^{2} \leq 2 t_{0}$, so with Step 1 we obtain

$$
1 \wedge \bar{\sigma}^{2} \mathcal{E} \leq C(\kappa) t_{0}
$$

So there exists some $\tau>0$ such that if $t_{0}<\tau$, then

$$
\begin{aligned}
\frac{t_{0}}{4} \sup \left\{E^{\epsilon}(x, t) \mid(x, t) \in \mathcal{P}_{\sqrt{t_{0}} / 2}\left(x_{0}, t_{0}\right)\right\} & =f\left(\sqrt{t_{0}} / 2\right) \\
& \leq f\left(\sigma_{0}\right) \\
& =(2 \bar{\sigma})^{2} \mathcal{E} \\
& \leq C t_{0}
\end{aligned}
$$

In particular, $E^{\epsilon}\left(x_{0}, t_{0}\right) \leq C$ for $t_{0} \leq \tau$.

Next define

$$
\alpha_{\infty}^{\epsilon}\left(t ; x_{0}, t_{0}\right):=\int \rho\left(x_{0}-x, t_{0}-t\right) d \mu_{t}^{\epsilon}(x) .
$$

Vol. $16, n^{\circ} 4-1999$. 
PRoposirion 5.2. - Suppose that $u^{\epsilon}$ is a solution of (1.2) on $\mathbf{R}^{d} \times\left[T_{0}, T_{1}\right]$, with $\epsilon \leq 1$, and that for all $s \leq 1$ and all $(x, t) \in \mathbf{R}^{d} \times\left[T_{0}, T_{1}\right]$ we have

$$
\mu_{t}^{\epsilon}\left(B_{s}(x)\right) \leq \kappa s^{d-p} .
$$

Then there are constants $\eta, \rho_{0}, C$ which may depend on $\kappa$, such that if

$$
\alpha_{\infty}^{\epsilon}\left(t_{0}-\tau^{2} ; x_{0}, t_{0}\right) \leq \eta
$$

for some $\tau>0$ and $\left(x_{0}, t_{0}\right) \in B_{r / 4} \times\left[T_{0}+\tau^{2}, T_{1}\right]$, then we have

$$
E^{\epsilon}(x, t) \leq \frac{C}{\left(\rho_{0} \tau\right)^{p}} \quad \text { in } \mathcal{P}_{p_{0} \tau}\left(x_{0}, t_{0}\right)
$$

Remark. - The point is that when we omit the cutoff function $\gamma$ from the integral, we no longer require $\tau$ to be small.

Proof. - The assumption that $\tau$ is small is used only in two places in the proof of Theorem 4.1 The first is in deriving (4.4), where it is used to guarantee that $\gamma$ is nonzero on a certain set. This clearly is no longer necesary when using $\alpha_{\infty}^{\epsilon}$ instead of $\alpha_{r}^{\epsilon}$.

Second, in (4.5) we employ the monotonicity formula Lemma 4.1, and thereby pick up an error term which is bounded by $\kappa \tau^{2}$. If however we work with $\alpha_{\infty}^{\epsilon}$ instead of $\alpha_{r}^{\epsilon}$, then there is no error term in the monotonicity formula. Indeed, setting $\gamma \equiv 1$ in (4.1) we obtain

$$
\frac{d}{d t} \alpha_{\infty}^{\epsilon}\left(t ; x_{0}, t_{0}\right) \leq 0
$$

Thus in this situation we can derive (4.5) with no restrictions on $\tau$.

\section{REGULARITY}

In this section we prove a uniform asymptotic regularity result for the usual $\mathbf{R}^{2}$-valued Ginzburg-Landau system

$$
u_{t}^{\epsilon}-\Delta u^{\epsilon}+\frac{1}{\epsilon^{2}}\left(\left|u^{\epsilon}\right|^{2}-1\right) u^{\epsilon}=0,
$$

in 2 space dimensions.

We will use the notation

$$
Q_{r}(\theta):=B_{\theta r}^{2} \times\left[(1-\theta) r^{2}, r^{2}\right]
$$


for $r>0, \theta \in(0,1]$. We set $Q_{r}:=Q_{r}(1)$ and $Q(\theta):=Q_{1}(\theta)$. For $(x, t) \in Q_{r}$, we define

$$
\delta(x, t)=(r-|x|) \wedge t
$$

To simplify notation, we do not explicitly indicate the dependence of $\delta$ on $r$. Note that $\delta$ is just the distance to the parabolic boundary of $Q_{r}$.

Our main result is

THEOREM 6.1. - Suppose that $u^{\epsilon}$ solves (6.1) in $Q_{r}$ with

$$
\mu_{t}^{\epsilon}\left(B_{r}\right) \leq \kappa
$$

for all $0 \leq t \leq r^{2}$. Then

$$
\delta(x, t)^{2} E^{\epsilon}(x, t) \leq C(\kappa) \quad \text { in } Q_{r}(\theta) .
$$

In particular, for every $\theta<1$,

$$
E^{\epsilon} \leq C(\kappa, \theta) \quad \text { in } Q_{r}(\theta) \text {. }
$$

Combining this with the short-time regularity result, Proposition 5.1, we immediately deduce the following

COROLlary 6.1. - Suppose that $u^{\epsilon}$ satisfies the hypothesis of Theorem 6.1. If, in addition,

$$
E^{\epsilon}(x, 0) \leq \kappa \quad \text { for all } x \in B_{r}
$$

then

$$
E^{\epsilon}(x, t) \leq C(\kappa) \quad \text { for all }(x, t) \in B_{r / 2} \times\left[0, r^{2}\right]
$$

We start by proving a compactness result that we will use several times.

LEMMA 6.1. - Suppose that $\left\{v_{n}\right\}$ are functions such that for each $n$,

$$
v_{n, t}-\Delta v_{n}=\frac{1}{\epsilon_{n}^{2}}\left(1-\left|v_{n}\right|^{2}\right) v_{n}
$$

on $Q_{r}$, for some $\epsilon_{n}>0$. Let $E_{n}=e_{\epsilon_{n}}\left(v_{n}\right)$. If $E_{n} \leq C$ and

$$
\sup _{t \in\left[0, r^{2}\right]} \int_{B_{r}} E_{n}(x, t) d x \leq C
$$

Vol. 16, $\mathrm{n}^{\circ}$ 4-1999. 
uniformly in $n$, then $v_{n}$ is locally uniformly continuous (Lipschitz in the $x$ variables and $C^{0,1 / 4}$ is the t-variable), and $E_{n}$ is precompact in $L_{\mathrm{loc}}^{2}\left(Q_{r}\right)$. Moreover, if $\epsilon_{n} \rightarrow \bar{\epsilon} \in[0,+\infty]$ and

$$
v_{n} \rightarrow \bar{v} \text { locally uniformly, } \quad E_{n} \rightarrow \bar{E} \quad \text { in } L_{\mathrm{loc}}^{2},
$$

then

(i) if $\bar{\epsilon}=0$, then $\bar{v}$ solves

$$
\bar{v}_{t}-\Delta \bar{v}=|\nabla \bar{v}|^{2} \bar{v}
$$

and $\bar{E}=|\nabla \bar{v}|^{2} / 2$.

(ii) if $\bar{\epsilon} \in(0, \infty)$. then $\bar{v}$ solves

$$
\bar{v}_{+}-\Delta \bar{v}=\frac{1}{\bar{\epsilon}^{2}}\left(1-|\bar{v}|^{2}\right) \bar{v}
$$

and $\bar{E}=e_{\bar{\epsilon}}(\bar{v})$.

(iii) if $\bar{\epsilon}=+\infty$, then $\bar{v}$ solves

$$
\bar{v}_{t}-\Delta \bar{v}=0
$$

and $\bar{E}=|\nabla \bar{v}|^{2} / 2$. Finally, $E_{n}(\cdot, t) \rightarrow \bar{E}(\cdot, t)$ weakly in $L_{\mathrm{loc}}^{2}\left(B_{r}\right)$ for every $0<t<r^{2}$.

Proof: - 1. The proof does not in any way depend of the radius $r$, so we work on $Q$ to simplify notation. It is obvious that $v_{n}$ and $\nabla v_{n}$ are uniformly bounded in $L_{\infty}$. Let $Q^{\prime}:=Q\left(\theta_{1}\right)$ for some $\theta_{1}<1$, and fix a function $\zeta \in C_{0}^{\infty}(Q)$ such that $\zeta \equiv 1$ on $Q^{\prime}$.

By using $\zeta$ in the estimate (2.3) we immediately obtain

$$
\iint_{Q^{\prime}}\left|v_{n, t}\right|^{2} d x d t \leq C\left(Q^{\prime}\right) .
$$

2. Now we define $Q^{\prime \prime}:=Q\left(\theta_{2}\right)$ for some $\theta_{2}<\theta_{1}$. From Step 1 we know that $v_{n}(\cdot, t)$ is Lipschitz in $B_{r}$ for every $t \in\left[0, r^{2}\right]$. Fix $\left(x, t_{1}\right),\left(x, t_{2}\right) \in Q^{\prime \prime}$ with $t_{1}<t_{2}$. For all $s$ such that $B_{s}(x) \subset B_{\theta_{1} r}$, we may integrate the inequality

$$
\left|v_{n}\left(x, t_{2}\right)-v_{n}\left(x, t_{1}\right)\right| \leq\left|v_{n}\left(y, t_{2}\right)-v_{n}\left(y, t_{1}\right)\right|+C|x-y|
$$


over $y \in B_{s}(x)$ to obtain

$$
\begin{aligned}
\left|v_{n}\left(x, t_{2}\right)-v_{n}\left(x, t_{1}\right)\right| & \leq \frac{1}{\pi s^{2}} \int_{B_{s}(x)}\left[v_{n}\left(y, t_{2}\right)-v_{n}\left(y, t_{1}\right)\right] d y+C s \\
& =\frac{1}{\pi s^{2}} \int_{t_{1}}^{t_{2}} \int_{B_{s}(x)} v_{n, t}(y, s) d y d s+C s \\
& \leq \frac{C}{s} \sqrt{t_{2}-t_{1}}\left(\int_{t_{1}}^{t_{2}} \int_{B_{s}(x)} v_{n},_{t}^{2}(y, s) d y d s\right)^{1 / 2}+C s \\
& \leq \frac{C}{s} \sqrt{t_{2}-t_{1}}+C s .
\end{aligned}
$$

In the final inequality we have used (6.7). If $t_{2}-t_{1}$ is sufficiently small, then $B_{s}(x) \subset B_{\theta_{1} r}$ for $s=\left(t_{2}-t_{1}\right)^{1 / 4}$. The above inequality with this value of $s$ then yields

$$
\left|v_{n}\left(x, t_{2}\right)-v_{n}\left(x, t_{1}\right)\right| \leq C\left(Q^{\prime \prime}\right)\left(t_{2}-t_{1}\right)^{1 / 4} .
$$

This implies that $v_{n}$ is locally uniformly continuous, as claimed.

3. We multiply (2.6) by $\zeta^{2} E_{n}$ and integrate over $Q$ to find that

$$
\begin{aligned}
\iint_{Q} \zeta^{2}\left[\left|\nabla E_{n}\right|^{2}+\right. & \left.E_{n}\left|\nabla^{2} v_{n}\right|^{2}+E_{n} \frac{1}{2 \epsilon_{n}^{2}}\left(1-\left|v_{n}\right|^{2}\right)^{2}\left|v_{n}\right|^{2}\right] d x d t \\
& \leq \iint_{Q}\left(C \zeta^{2} E_{n}^{3}+\zeta \zeta_{t} E_{n}^{2}-2 \zeta E_{n} \nabla \zeta \cdot \nabla E_{n}\right) d x d t
\end{aligned}
$$

We now use Cauchy's inequality to cancel the bad terms on the right-hand side against terms on the left, thus leaving us with

$$
\begin{aligned}
\iint_{Q^{\prime}} E_{n}\left|\nabla^{2} v_{n}\right|^{2}+\left|\nabla E_{n}\right|^{2}+E_{n} & \frac{\left(1-\left|v_{n}\right|^{2}\right)^{2}}{\epsilon_{n}^{4}}\left|v_{n}\right|^{2} d x d t \\
& \leq C \iint_{Q}\left(\zeta^{2} E_{n}^{3}+\zeta E_{n}^{2}\right) d x d t
\end{aligned}
$$

In particular, $\nabla E_{n}$ is uniformly bounded in $L^{2}\left(Q^{\prime}\right)$.

4. We next claim that

$$
\iint_{Q^{\prime}} \frac{\left(1-\left|v_{n}\right|^{2}\right)^{2}}{\epsilon_{n}^{4}} d x d t \leq C .
$$

Multiplying (2.6) by $\zeta^{2}$ and calculating as in Step 3, we find that

$$
\iint_{Q^{\prime}} \frac{\left(1-\left|v_{n}\right|^{2}\right)^{2}}{\epsilon_{n}^{4}}\left|v_{n}\right|^{2} d x d t \leq C .
$$

Vol. $16, \mathrm{n}^{\circ} 4-1999$. 
Because $E_{n}=e_{\epsilon_{n}}\left(v_{n}\right)$ is uniformly bounded, there exists some $\bar{\epsilon}>0$ such that $\left|v_{n}\right| \geq 1 / 2$ whenever $\epsilon_{n} \leq \bar{\epsilon}$, so that the above estimate plainly implies (6.9) in this case. On the other hand, if $\epsilon_{n} \geq \bar{\epsilon}$, then

$$
\iint_{Q^{\prime}} \frac{\left(1-\left|v_{n}\right|^{2}\right)^{2}}{\epsilon_{n}^{4}} d x d \tau \leq \frac{1}{\bar{\epsilon}^{2}} \iint_{Q} E_{n} d x d \tau \leq C .
$$

5. Fix a smooth function $\eta$ supported in $Q^{\prime}$ such that $\eta \equiv 1$ on $Q^{\prime \prime}$. By differentiating (6.1) with respect to time and multiplying by $2 v_{n, t}$, we obtain $\frac{d}{d t}\left|v_{n, t}\right|^{2}-\Delta\left|v_{n, t}\right|^{2}+2\left|\nabla v_{n, t}\right|^{2}+\frac{2}{\epsilon_{n}^{2}}\left[\left(\left|v_{n}\right|^{2}-1\right)\left|v_{n, t}\right|^{2}+2\left|v_{n} \cdot v_{n, t}\right|^{2}\right]=0$

Multiplying by $\eta$ and integrating, we get

$$
\begin{aligned}
& \left.\int_{B_{R}} \eta\left|v_{n}, t\right|^{2} d x\right|_{t=T}+2 \iint_{Q^{\prime}} \eta\left|\nabla v_{n}, t\right|^{2} d x d t \\
& \leq \iint_{Q^{\prime}} 2 \eta \frac{1-\left|v_{n}\right|^{2}}{\epsilon_{n}^{2}}\left|v_{n, t}\right|^{2}+\left(\eta_{t}+\Delta \eta\right)\left|v_{n, t}\right|^{2} d x d t .
\end{aligned}
$$

In particular, using Step 1, Step 4, and Cauchy's inequality we obtain

$$
\iint_{Q^{\prime}} \eta\left|\nabla v_{n, t}\right|^{2} d x d t \leq C(\delta)+\delta \iint \eta^{2}\left|v_{n}, t\right|^{4} d x d t
$$

where $\delta>0$ will be selected below. Defining $\xi=\eta^{1 / 2}$, we use a Sobolev-Poincaré inequality to compute

$$
\begin{aligned}
\iint_{Q^{\prime}} \eta^{2}\left|v_{n, t}\right|^{4} d x d t & =\iint_{Q^{\prime}}\left|\xi v_{n, t}\right|^{4} d x d t \\
& \leq C \iint_{Q^{\prime}}\left|\nabla\left(\xi v_{n, t}\right)\right|^{2} d x d t \\
& \leq C \iint_{Q^{\prime}} \eta\left|\nabla v_{n, t}\right|^{2}+|\nabla \xi|^{2}\left|v_{n, t}\right|^{2} d x d t .
\end{aligned}
$$

We substitute this into (6.11), select $\delta$ small and use Step 1 to conclude that

$$
\iint_{Q^{\prime \prime}}\left|\nabla v_{n, t}\right|^{2} d x d t \leq C\left(Q^{\prime \prime}\right)
$$

Since

$$
E_{n, t} \leq\left|\nabla v_{n}\right|\left|\nabla v_{n, t}\right|+\frac{\left(1-\left|v_{n}\right|^{2}\right)}{\epsilon_{n}^{2}}\left|v_{n, t}\right|,
$$

we easily deduce that $E_{n, t}$ is uniformly bounded in $L^{2}\left(Q^{\prime \prime}\right)$. With Step 3 this proves that $\left\{E_{n}\right\}$ is precompact in $L_{\text {loc }}^{2}(Q)$. 
In fact, the above estimates show something slightly stronger: that $\left\{\left|\nabla v_{n}\right|^{2}\right\}$ and $\left\{\left(\left|v_{n}\right|^{2}-1\right)^{2} / \epsilon_{n}^{2}\right\}$ are both precompact in $L_{\text {loc }}^{2}(Q)$.

6. Assume now that (6.3) holds. We first consider the case $\bar{\epsilon}=0$. In this case, $\rho_{n}:=\left|v_{n}\right| \rightarrow 1$ uniformly as $n \rightarrow \infty$, so for $n$ sufficiently large we may define a globally single-valued function $\phi_{n}$ such that

$$
v_{n}=\rho_{n}\left(\cos \left(\phi_{n}\right), \sin \left(\phi_{n}\right)\right) \text {. }
$$

We normalize $\phi_{n}$ by imposing the requirement $\phi_{n}(0) \in[0,2 \pi)$. We then see from (6.3) that $\phi_{n} \rightarrow \bar{\phi}$ locally uniformly in $Q$, where $\bar{\phi}$ satisfies $\bar{v}=(\cos (\bar{\phi}), \sin (\bar{\phi}))$. It is also clear that $\nabla \phi_{n} \rightarrow \nabla \bar{\phi}$ weakly in $L^{2}$. Writing (6.1) in terms of $\rho_{n}$ and $\phi_{n}$, we obtain two equations, one of which is

$$
\rho_{n}^{2} \phi_{n, t}-\operatorname{div}\left(\rho_{n}^{2} \nabla \phi_{n}\right)=0
$$

With the above estimates, we may pass to limits to find that $\bar{\phi}$ satisfies

$$
\bar{\phi}_{t}-\Delta \bar{\phi}=0
$$

Rewriting this in terms of $\bar{v}$ yields (6.4).

7. Still assuming that $\bar{\epsilon}=0$, we need to prove that $\bar{E}=(1 / 2)|\nabla \bar{v}|^{2}$. In view of the results of Step 5 , it suffices to show that

$$
\frac{\left(\left|v_{n}\right|^{2}-1\right)^{2}}{\epsilon_{n}^{2}} \rightarrow 0
$$

in $L_{\text {loc }}^{2}(Q)$. From (6.8) and the fact that $\left|v_{n}\right| \rightarrow 1$ uniformly, we have

$$
\iint_{Q^{\prime}} \frac{\left(1-\left|v_{n}\right|^{2}\right)^{4}}{\epsilon_{n}^{6}} d x d t \leq C\left(Q^{\prime}\right)
$$

for $n$ sufficiently large. This immediately gives the desired conclusion.

8. If we assume that $\bar{\epsilon} \in(0, \infty)$ or $\bar{\epsilon}=+\infty$, the stated conclusions follow in a straightforward fashion from the estimates of Steps 3 and 5.

9. Finally, fix some $0<t<1$ and let $\phi \in L^{2}\left(B_{1}\right)$. Suppose also that $\phi$ is supported in $U \subset \subset B_{1}$. Fix $h>0$ small enough that $t+h<1$. Then

$$
\int\left[E_{n}^{\prime}(x, t)-\overline{E^{\prime}}(x, t)\right] \phi(x) d x=I_{1}+I_{2}+I_{3},
$$

where

$$
I_{1}:=\frac{1}{h} \int_{t}^{t+h} \int\left[E_{n}(x, s)-\bar{E}(x, s)\right] \phi(x) d x d s
$$




$$
I_{2}:=\frac{1}{h} \int_{t}^{t+h} \int\left[E_{n}(x, t)-E_{n}(x, s)\right] \phi(x) d x d s,
$$

and $I_{3}$ has the same form as $I_{2}$. It is clear that $I_{1} \rightarrow 0$ as $n \rightarrow \infty$. Also,

$$
\begin{aligned}
I_{2} & =\frac{1}{h} \int_{t}^{t+h} \iint_{s}^{t} E_{n, t}(x, r) d r d x d s \\
& =\frac{1}{h} \int_{t}^{t+h} \int(t+h-r) E_{n, t}(x, r) d x d r
\end{aligned}
$$

by Fubini's Theorem. Thus

$$
\begin{aligned}
I_{2} & \leq \frac{1}{h}\left(\int_{t}^{t+h} \int_{U} \phi(x)^{2}(t+h-r)^{2} d x d r\right)^{1 / 2}\left(\int_{t}^{t+h} \int_{U} E_{n, t}^{2} d x d r\right)^{1 / 2} \\
& \leq C \sqrt{h}\|\phi\|_{L^{2}}
\end{aligned}
$$

using the fact that $E_{n, t}$ is locally uniformly bounded in $L^{2}$. Clearly $I_{3}$ satisfies a similar estimate, so

$$
\limsup _{n} \int\left[E_{n}(x, t)-\bar{E}(x, t)\right] \phi(x) d x \leq C \sqrt{h} .
$$

Since $h$ is arbitrary, we are finished.

We are now ready for the

Proof of Theorem 6.1.

1. First note that the statement of the theorem is invariant under rescalings, so we may assume without loss of generality that $r=1$.

Suppose that

$$
\sup \left\{\delta(x, t)^{2} E^{\epsilon}(x, t) \mid \epsilon>0,(x, t) \in Q^{\prime}\right\}=+\infty .
$$

Temporarily fix $n \geq \eta^{-1 / 2}$, where $\eta$ is the constant from Theorem 4.1. For each $\epsilon>0$, the function

$$
(x, t) \mapsto \alpha_{\delta(x, t)}^{\epsilon}\left(t-\frac{\delta^{2}(x, t)}{n^{2}} ; x, t\right)
$$

is well-defined and continuous in $Q$ and approaches 0 as $\delta(x, t)$ approaches 0 . From (6.2) it is clear that the hypotheses of Theorem 4.1 are satisfied. It follows that

$$
\limsup _{\epsilon \rightarrow 0} \sup _{(x, t) \in Q} \alpha_{\delta(x, t)}^{\epsilon}\left(t-\frac{\delta^{2}(x, t)}{n^{2}} ; x, t\right)>\eta
$$


otherwise (6.12) and Theorem 4.1 would lead to a contradiction. Thus we can select $\epsilon_{n} \rightarrow 0,\left(x_{n}, t_{n}\right) \in Q$ such that

$$
\alpha_{\delta\left(x_{n}, t_{n}\right)}^{\epsilon_{n}}\left(t_{n}-\frac{\delta^{2}\left(x_{n}, t_{n}\right)}{n^{2}} ; x_{n}, t_{n}\right)=\eta / 2
$$

and for all $(x, t) \in Q$ such that $t \leq t_{n}$, we have

$$
\alpha_{\delta(x, t)}^{\epsilon_{n}}\left(t-\frac{\delta^{2}(x, t)}{n^{2}} ; x, t\right) \leq \eta / 2 .
$$

The small-energy regularity Theorem 4.1 now implies that

$$
F_{1}^{\epsilon_{n}}(x, t) \leq C n^{2} / \delta^{2}(x, t)
$$

for all $(x, t) \in Q$ such that $t \leq t_{n}$. In particular, if we let $r_{n}=\delta\left(x_{n}, t_{n}\right) / n$, then

$$
E^{\epsilon_{n}}(x, t) \leq C / r_{n}^{2} \quad \text { in } \mathcal{P}_{\frac{1}{2} \delta\left(x_{n}, t_{n}\right)}\left(x_{n}, t_{n}\right)
$$

2. For $(x, t) \in \mathcal{P}_{n}(0,0)$ let $y_{n}(x):=x_{n}+r_{n} x$ and $s_{n}:=t_{n}+r_{n}^{2} t$, and define

$$
v_{n}(x, t)=u^{\epsilon_{n}}\left(y_{n}, s_{n}\right)
$$

Note that $\left(y_{n}, s_{n}\right) \in \mathcal{P}_{\delta\left(x_{n}, t_{n}\right)}\left(x_{n}, t_{n}\right)$ for $(x, t) \in \mathcal{P}_{n}(0,0)$, so $v_{n}$ is well-defined on this set. Let $\tilde{\epsilon}_{n}-\epsilon_{n} / r_{n}$. Then $v_{n}$ solves

$$
v_{n, t}-\Delta v_{n}+\frac{1}{\tilde{\epsilon}_{n}^{2}}\left(\left|v_{n}\right|^{2}-1\right) v_{n}=0 .
$$

If we denote $\tilde{E}_{n}(x, t)=e_{\tilde{\epsilon}_{n}}\left(v_{n}\right)$, then by rescaling equations (6.15), (6.2), and (6.13) respectively, we obtain

$$
\begin{gathered}
\tilde{E}_{n} \leq C \quad \text { in } \mathcal{P}_{n / 2}(0,0), \\
\int_{B_{R}} \tilde{E}_{n}(x, t) d x \leq K \\
\int \gamma\left(\frac{|x|}{n}\right) \rho(x, 1) \tilde{E}_{n}(x,-1) d x=\eta / 2
\end{gathered}
$$

whenever $R \leq\left(4 r_{n}\right)^{-1},(x, t) \in \mathcal{P}_{n / 2}(0,0)$. These estimates are all independent of $n$. 
3. If $(x, t) \in \mathcal{P}_{\sqrt{n}}(0,0)$, let $\tau_{n}(x, t):=\delta\left(y_{n}(x), s_{n}(t)\right) / \delta\left(x_{n}, t_{n}\right)$. Rescaling (6.14) then yields

$$
\int \gamma\left(\frac{|x-y|}{n \tau_{n}(x, t)}\right) \rho\left(x-y, \tau_{n}^{2}\right) \tilde{E}_{n}\left(y, t-\tau_{n}^{2}\right) d y \leq \eta / 2 .
$$

Also, if $(x, t) \in \mathcal{P}_{\sqrt{n}}(0,0)$, it is clear that $\left(y_{n}(x), s_{n}(t)\right) \in$ $\mathcal{P}_{\delta\left(x_{n}, t_{n}\right) / \sqrt{n}}\left(x_{n}, t_{n}\right)$, and so $\tau_{n}(x, t) \leq 1$. Moreover, if $|x| \leq \sqrt{n}$,

$1-\left|y_{n}(x)\right| \geq 1-\left|x_{n}\right|-\delta\left(x_{n}, t_{n}\right) / \sqrt{n} \geq \delta\left(x_{n}, t_{n}\right)\left(1-n^{-1 / 2}\right)$.

Similarly $s_{n}(t) \geq \delta^{2}\left(x_{n}, t_{n}\right)\left(1-n^{-1}\right)$ whenever $t \in[-n, 0]$. Thus, recalling the definition of $\delta$,

$$
\sup \left\{\left|\tau_{n}(x, t)-1\right|:(x, t) \in \mathcal{P}_{\sqrt{n}}(0,0)\right\} \rightarrow 0 \quad \text { as } n \rightarrow \infty
$$

4. We may assume after passing to a further subsequence that $\epsilon_{n} \rightarrow L \in$ $[0,+\infty]$ and, as a result of Lemma 6.1 , that $v_{n} \rightarrow \bar{v}$ locally uniformly and that $E_{n}(\cdot, t) \rightarrow \bar{E}(\cdot, t)$ weakly in $L_{\text {loc }}^{2}\left(\mathbf{R}^{2}\right)$, for every $t<0$..

Let

$$
\bar{\alpha}\left(t ; x_{0}, t_{0}\right):=\int \rho\left(x_{0}-x, t_{0}-t\right) \bar{E}(x, t) d x .
$$

$\bar{E}$ inherits the estimates

$$
\begin{gathered}
\|\bar{E}\|_{L^{\infty}\left(\mathcal{P}_{\infty}\right)} \leq C, \\
\|\bar{E}(\cdot, t)\|_{L^{1}\left(\mathbf{R}^{2}\right)} \leq K \quad \text { for every } t \leq 0
\end{gathered}
$$

from (6.16) and (6.17) respectively, and

$$
\bar{\alpha}(-1 ; 0,0)=\eta / 2
$$

from (6.18) Also, (6.19), (6.20) and Fatou's lemma imply that

$$
\bar{\alpha}(t-1 ; x, t) \leq \eta / 2
$$

for all $(x, t) \in \mathcal{P}_{\infty}$.

5. We now consider three cases, corresponding to the three cases of Lemma 6.1. In each case we will show that $\bar{v}$ must be a constant, in contradiction to (6.23).

Case (i): $\bar{\epsilon}=0$. 
From the proof of Lemma 6.1, we see that we may write $\bar{v}=$ $(\cos (\bar{\phi}), \sin (\bar{\phi}))$, where $\bar{\phi}$ solves the heat equation on $\mathcal{P}_{\infty}$, and that $\bar{E}=|\nabla \bar{\phi}| / 2 \leq C$. It follows that $\nabla \bar{\phi}$ also solves the heat equation on $\mathcal{P}_{\infty}$. Standard Liouville-type theorems then imply that $\nabla \bar{\phi}$ is constant, and we see from (6.22) that this constant can only equal zero. This contradicts (6.23).

Case (ii): $\bar{\epsilon} \in(0,+\infty)$.

Lemma 6.1 implies that $\bar{v}$ solves (6.5) on $\mathcal{P}_{\infty}$. As in case (i), a Liouvilletype theorem shows that $\bar{v}$ must be constant. This is the content of Theorem 7.1, which is proved in the next section.

Case (iii): $\bar{\epsilon}=+\infty$.

By Lemma 6.1, $\bar{v}$ solves the heat equation on $\mathcal{P}_{\infty}$. An argument very similar to that given for case (i) above shows that $\bar{v}$ is constant. Thus the proof is complete.

Remark. - 1. Heuristically, case (i) corresponds to the possibility that a singularity might form in the interior of $Q$, and cases (ii) and (iii) correspond to the possibility that a singularity might enter $Q$ at the boundary. Thus they arise as a consequence of the fact that the theorem is local in nature.

2. Carrying out a similar blowup argument for the usual Ginzburg-Landau system (1.1) in $d$ space dimensions, one may obtain a function $\bar{v}$ solving either (6.4), (6.5), or (6.6) on $\mathbf{R}^{d} \times(-\infty, 0]$ and satisfying the estimates (6.21), (6.23), (6.24), and

$$
\int_{B_{R}\left(x_{0}\right)} \bar{E}\left(x, t_{0}\right) d x \leq C R^{d-2}
$$

for every $\left(x_{0}, t_{0}\right) \in \mathbf{R}^{d} \times(-\infty, 0]$. To establish a regularity result like the one given above, one would need a Liouville-type theorem asserting that such a function is necessarily constant. We conjecture that such a Liouville-type theorem holds.

3. For the generalized Ginzburg-Landau system (1.2), one may again carry out a blowup argument to find a function solving a limiting PDE on the set $\mathbf{R}^{d} \times(-\infty, 0]$, but one does not expect a Liouville-type theorem to hold except under special circumstances. In this case, these results can be interpreted as giving some qualitative information about the types of singularities that can occur, as in Struwe [25].

\section{A LIOUVILLE-TYPE THEOREM}

We start out with a Liouville-type theorem for the elliptic GinzburgLandau equation in $\mathbf{R}^{2}$. Stronger results of the same character may be Vol. $16, \mathrm{n}^{\circ} 4-1999$. 
found in Brezis, Merle and Riviere [6]; we include this here for the reader's convenience.

LeMma 7.1. - Suppose that $u: \mathbf{R}^{2} \rightarrow \mathbf{R}^{2}$ solves

$$
-\Delta u+\left(|u|^{2}-1\right) u=0
$$

and that $E(x):=(1 / 2)|\nabla u|^{2}+W(u)$ is bounded and satisfies

$$
\int_{\mathbf{R}^{2}} E(x) d x \leq C .
$$

Then $u$ is constant.

Proof. - As in (2.3) we derive

$$
\int\left[\Delta \eta(x) E(x)-\nabla^{2} \eta(x) \nabla u(x) \cdot \nabla u(x)\right] d x=0 .
$$

for smooth test functions $\eta$. Let $\eta(x)=g(|x|)$, where

$$
g(s)= \begin{cases}s^{2} & \text { if } s \leq r \\ 2 r^{2} & \text { if } s \geq 2 r\end{cases}
$$

with $0 \leq g^{\prime}(s), g^{\prime \prime}(s) \geq-C / r$ for $s \in[r, 2 r]$. Then $\left|\nabla^{2} \eta(x)\right| \leq C / r$ if $r \leq|x| \leq 2 r$, so

$$
\int_{B_{r}} W(u(x)) d x \leq \frac{C}{r} \int_{\{r \leq|x| \leq 2 r\}} E(x) d x .
$$

Letting $r \rightarrow \infty$ and using (7.1), the right-hand side tends to zero. Thus $|u| \equiv 1$, and so the equation becomes $\Delta u=0$. Now the result follows from the standard Liouville's Theorem.

With this theorem we complete the proof of Theorem 6.1 .

THEOREM 7.1. - Let $u$ be a function solving

$$
u_{t}-\Delta u+\frac{1}{\bar{\epsilon}^{2}}\left(|u|^{2}-1\right) u=0
$$

on $\mathcal{P}_{\infty}=\mathbf{R}^{2} \times(-\infty, 0]$ and satisfying in addition the estimates

$$
\begin{gathered}
|u| \leq 1, \quad\|E\|_{L^{\infty}(\mathcal{P} \infty)} \leq \kappa, \\
\|E(\cdot, t)\|_{L^{1}\left(\mathbf{R}^{2}\right)} \leq \kappa \quad \text { for all } t \leq 0
\end{gathered}
$$




$$
\alpha(t-1 ; x, t):=\int \rho(x-y, 1) E(y, t) d y \leq \eta / 2,
$$

where $E:=e_{\bar{\epsilon}}(u), \eta$ is the number from Proposition 5.2, and $\kappa$ may be any positive number.

Then $u$ is constant.

Proof. -1 . We may assume by rescaling that $\bar{\epsilon}=1$.

For $R>0, T \leq 0$ set

$$
H(R ; T):=\sup \left\{\alpha\left(t-R^{2} ; x, t\right) \mid x \in \mathbf{R}^{2}, t \in(-\infty, T]\right\}
$$

Then (7.5) states that

$$
H(1 ; T) \leq \eta / 2
$$

for all $T \leq 0$.

2. If there is some $T<0$ for which

$$
\sup _{R \geq 1} H(R ; T) \leq \eta,
$$

then for every $x \in \mathbf{R}^{2}, t \leq T, R \geq 1$ we have the estimate

$$
\alpha\left(t-R^{2} ; x, t\right) \leq \eta
$$

and Proposition 5.2 implies that

$$
E(x, t) \leq C / R^{2}
$$

Letting $R \rightarrow \infty$, we find that $\bar{E}(x, t)=0$ for all $x \in \mathbf{R}^{2}, t \leq T$. This implies that $u$ is constant on $\{t \leq T\}$, which in turn implies the conclusion of the lemma.

3. In order to demonstrate that (7.7) holds and complete the proof, we assume that

$$
\sup _{R \geq 1} H(R ; T)>\eta
$$

for every $T<0$, toward an eventual contradiction. We first claim that with (7.6) this implies that for each $T<0$, there exists some $R(T)>1$ such that $H(R(T), T)=\eta$.

Vol. 16, $n^{\circ}$ 4-1999. 
To prove this, it suffices to show that for each $T<0, H(\cdot, T)$ is locally Lipschitz in $[0, \infty)$. By the chain rule and (2.3), we have

$$
\begin{aligned}
\frac{d}{d R} \alpha\left(t-R^{2} ; x, t\right) \leq 2 R & \int \rho\left(x-y, R^{2}\right)\left|u_{t}\left(y, t-R^{2}\right)\right|^{2} d y \\
& +C(R) \int E\left(y, \iota-R^{2}\right) d y
\end{aligned}
$$

where $C(R)$ contains a factor of $2 R$ from the chain rule and sup norms of derivatives of $\rho$. One easily checks that $C(R)$ may be taken to be continuous. Now using estimate (6.10) with $\eta \equiv 1$ we get

$$
\int\left|u_{t}(y, t)\right|^{2} d y \leq \int_{-\infty}^{t} \int\left|u_{t}\right|^{2} d x d t \leq \sup _{s \leq t}\|E(\cdot, s)\|_{L^{1}},
$$

where we have used (2.3) in the last inequality. With (7.4) we find that

$$
\frac{d}{d R} \alpha\left(t-R^{2} ; x, t\right) \leq C(R)
$$

which implies that $\frac{d}{d R} H(R, T) \leq C(R)$ for all $T<0$.

4. From (7.4) it is clear that for each $x \in \mathbf{R}^{2}$ and $t<0$,

$$
\lim _{R \rightarrow \infty} \alpha\left(t-R^{2} ; x, t\right)=0
$$

Using this fact and Step 3, we may thus choose $x_{n} \in \mathbf{R}^{2}, t_{n} \leq-n$ such that for $R_{n}:=R(-n) \geq 1$,

$$
\alpha\left(t_{n}-R_{n} ; x_{n}, t_{n}\right)=3 \eta / 4
$$

Define

$$
u_{n}(x, t):=u\left(x_{n}+R_{n} x, t_{n}+R_{n}^{2} t\right), \quad E_{n}(x, t):=e_{1 / R_{n}}\left(u_{n}\right)(x, t)
$$

for $(x, t) \in \mathcal{P}_{\infty}$. Then rescaling as usual, we find that $u_{n}$ solves

$$
u_{n, t}-\Delta u_{n}+\frac{1}{R_{n}^{2}}\left(\left|u_{n}\right|^{2}-1\right) u_{n}-0
$$

with the estimates

$$
\left\|E_{n}(\cdot, t)\right\|_{L^{1}\left(\mathbf{R}^{2}\right)} \leq C,
$$


and for any $(x, t) \in \mathcal{P}_{\infty}$,

$$
\begin{aligned}
\alpha_{n}(t-1 ; x, t) & :=\int \rho(x-y, 1) E_{n}(y, t-1) d y \\
& =\alpha\left(\tilde{t}-R_{n}^{2} ; \tilde{x}, \tilde{t}\right) \\
& \leq H\left(R_{n} ; t_{n}\right)=\eta
\end{aligned}
$$

Here $(\tilde{x}, \tilde{t})=\left(R_{n} x+x_{n}, R_{n}^{2} t+t_{n}\right)$, so that $\tilde{t} \leq t_{n}$ for $t \leq 0$. Also,

$$
\alpha_{n}(1 ; 0,0)=\alpha\left(R_{n} ; x_{n}, t_{n}\right)=3 \eta / 4
$$

5. Now (7.9) and Proposition 5.2 imply that

$$
\sup \left\{E_{n}(x, t) \mid n \geq 1,(x, t) \in \mathcal{P}\right\} \leq C<+\infty .
$$

This shows that the hypotheses of the Compactness Lemma 6.1 are satisfied by the sequence $u_{n}$, with $\epsilon_{n}:=1 / R_{n} \leq 1$, so we may extract a subsequence converging to a function $\tilde{u}$ locally uniformly, with $E_{n} \rightarrow \tilde{E}$ in $L_{\text {loc }}^{2}\left(\mathcal{P}_{\infty}\right)$. The strong convergence of $E_{n}$ and (7.10) imply that

$$
\tilde{u} \quad \text { is not constant. }
$$

Since $\epsilon_{n} \leq 1$, we only need to consider two cases:

Case (i): $\epsilon_{n} \rightarrow 0$ and $\tilde{E}=\frac{1}{2}|\nabla \tilde{u}|^{2}$.

Then we show as in Step 5 of the proof of Theorem 6.1 that $\tilde{u}$ is constant, in contradiction to (7.11)

Case (ii): $\epsilon_{n} \rightarrow \tilde{\epsilon} \in(0,1]$ and $\tilde{E}=e_{\tilde{\epsilon}}(\tilde{u})$.

In this case, by rescaling we obtain

$$
\iint_{\mathcal{P}}\left|u_{n}, t\right|^{2} d x d t=\int_{-\infty}^{t_{n}} \int_{\mathbf{R}^{2}}\left|u_{t}\right|^{2} d x d t \rightarrow 0
$$

since $t_{n} \rightarrow-\infty$ as $n \rightarrow \infty$. With Lemma 6.1 this shows that $\tilde{u}$ solves the elliptic Ginzburg-Landau system

$$
-\Delta \tilde{u}+\frac{1}{\tilde{\epsilon}^{2}}\left(|\tilde{u}|^{2}-1\right) \tilde{u}=0
$$

and $\|\tilde{E}(\cdot, t)\|_{L^{1}\left(\mathbf{R}^{2}\right)}=\|\tilde{E}(\cdot)\|_{L^{1}\left(\mathbf{R}^{2}\right)} \leq C$. However, we have shown in Lemma 7.1 that that any such function must be constant, again contradicting (7.11).

Vol. 16, $n^{\circ}$ 4-1999. 


\section{REFERENCES}

[1] L. Ambrosio and H.M. Soner, Level set approach to mean curvature flow in arbitrary codimension. J. Diff. Geom., Vol. 43, 1996, pp. 693-737.

[2] G. Barles, H.M. Soner, and P.E. Souganidis, Front propagation and phase field theory. SIAM J. Cont. Opt., Vol. 31 (2), 1993, pp. 439-469.

[3] P. Bauman, C.N. Chen, D. Phillips, and P. Sternberg, Vortex annihilation in nonlinear heat flow for Ginzburg-Landau systems. European J. Appl. Math., Vol. 6(2), 1995, pp. $115-126$.

[4] F. Bethuel, H. Brezis, and F Hélein, Asymptotics for the minimization of a GinzburgLandau functional. Calc. Var., Vol. 1, 1993, pp. 123-148.

[5] F. BETHUEL, H. BREZIS, and F HÉlein, Ginzburg-Landau Vortices, Birkhäuser, Boston, 1994.

[6] H. BRezis, F. MERLE, and T. RIVIERE, Quantization effects for $-\Delta u=u\left(1-|u|^{2}\right)$ in $\mathbf{R}^{2}$ Archive Rat. Mech. Anal., Vol. 126, 1994, pp. 35-58.

[7] X. CHEN, Generation and propagation of the interface for reaction-diffusion equations Jour. Diff. Equations, Vol. 96, 1992, pp. 116-141.

[8] Y. Chen and M. Struwe, Existence and partial regularity results for the heat flow for harmonic maps. Math Z., Vol. 201, 1989, pp. 83-103.

[9] W. E., Dynamics of vortices in Ginzburg-Landau theories with applications to superconductivity. Physica D, Vol. 77, 1994, pp. 383-404.

[10] L.C. Evans, H.M. Soner, and P.E. Souganidis, Phase transitions and generalized motion by curvature. Comm. Pure Appl. Math., Vol. 65, 1992, pp. 1097-1123.

[11] T. ILMANEN, Convergence of the Allen-Cahn equation to Brakke's motion by mean curvature. J. Diff. Geom., Vol. 38 (2), 1993, pp. 417-461.

112] R.L. JerraRD, Fully nonlinear phase field equations and generalized mean curvature motion. Comm PDE, Vol. 20, 1995, pp. 233-265.

[13] R.L. JERRARD, Lower bounds for generalized Ginzburg-Landau functionals, center for Nonlinear Analysis Research Report No. 95-NA-020, 1995.

[14] R.L. JERRARD and H.M. SONER, Dynamics of Ginzburg-Landau vortices, Arch. Rat. Mech. Anal., Vol. 142, 1998, pp. 99-125.

[15] N.V. KRYLOV and M.V. SAFONOV, A certain property of solutions of parabolic equations with measurable coefficients. Math. USSR Izvestia, Vol. 16 (1), 1981, pp. 151-164.

[16] O.A. LAdYZhENSKya, V.A. SolonNikov, and N.N. Uraltseva. Linear and Quasilinear Equations of Parabolic Type, Amer. Math. Soc., Providence, RI, 1968.

[17] F.H. LIN, Solutions of Ginzburg-Landau equations and critical points of the renormalized energy. Ann. Inst. H. Poincaré Anal. Non Linéaire, Vol. 12 (5), 1995, pp. 599-622.

[18] F.H. Lin, Some dynamical properties of Ginzburg-Landau vortices. Comm. Pure Appl. Math., Vol. 49 (4), 1996, pp. 323-359.

[19] J.C. Neu, Vortices in complex scalar fields. Physica D, Vol. 43, 1990, pp. 385-406.

[20] L.M. Pismen and J. Rubinstein, Motion of vortex lines in the Ginzburg-Landau model. Physica D, Vol. 47, 1991, pp. 353-360.

[21] J. Rubinstein, Self-induced motion of line vortices. Quart. Appl. Math., Vol. 49 (1), 1991, pp. $1-9$.

[22] R. SCHOEN, Analytic aspects of the harmonic map problem, In S.S. Chern, editor, Seminar on Nonlinear Partial Differential Equations. Springer, Berlin, 1984.

[23] H. Mete Soner, Motion of a set by the curvature of its boundary. Jour. Diff. Equations, Vol. 101 (2), 1993, pp. 313-372.

[24] M. Struwe, On the evolution of harmonic maps in higher dimensions. J. Diff. Geom., Vol. 28, 1988, pp. 485-502.

[25] M. STRuwe. Variational Methods, Springer-Verlag, Berlin, 1990.

[26] M. STRUwE, On the asymptotic behavior of minimizers of the Ginzburg-Landau model in 2 dimensions. Diff. and Int. Equations, Vol. 7 (6), 1994, pp. 1613-1624.

[27] Y.KuRAmoto, Chemical Waves, Oscillations, and Turbulence, Springer-Verlag, Berlin, 1984.

(Manuscript received January 4 1996;

Revised version received April 2, 1997.) 